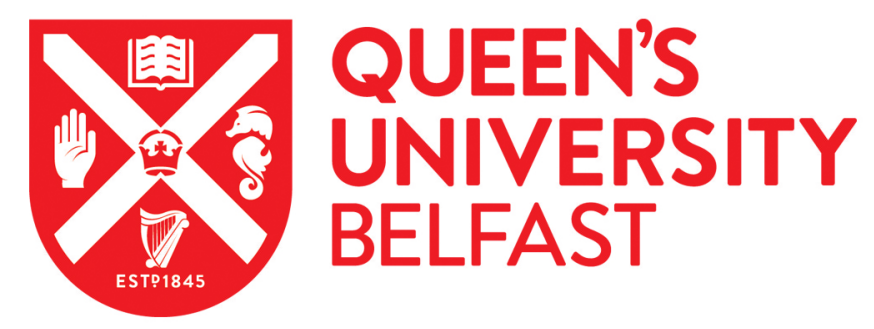

\title{
Tracing the Chromospheric and Coronal Magnetic Field with AIA, IRIS, IBIS, and ROSA Data
}

Aschwanden, M. J., Reardon, K., \& Jess, D. (2016). Tracing the Chromospheric and Coronal Magnetic Field with AIA, IRIS, IBIS, and ROSA Data. The Astrophysical Journal, 826(1), [61]. https://doi.org/10.3847/0004$637 \times / 826 / 1 / 61$

Published in:

The Astrophysical Journal

Document Version:

Publisher's PDF, also known as Version of record

Queen's University Belfast - Research Portal:

Link to publication record in Queen's University Belfast Research Portal

Publisher rights

(C) 2016 The American Astronomical Society. All rights reserved.

\section{General rights}

Copyright for the publications made accessible via the Queen's University Belfast Research Portal is retained by the author(s) and / or other copyright owners and it is a condition of accessing these publications that users recognise and abide by the legal requirements associated with these rights.

Take down policy

The Research Portal is Queen's institutional repository that provides access to Queen's research output. Every effort has been made to ensure that content in the Research Portal does not infringe any person's rights, or applicable UK laws. If you discover content in the Research Portal that you believe breaches copyright or violates any law, please contact openaccess@qub.ac.uk. 


\title{
TRACING THE CHROMOSPHERIC AND CORONAL MAGNETIC FIELD WITH AIA, IRIS, IBIS, AND ROSA DATA
}

\author{
Markus J. Aschwanden ${ }^{1}$, Kevin Reardon ${ }^{2,3,4}$, and Dave B. Jess ${ }^{4}$ \\ ${ }^{1}$ Lockheed Martin, Solar and Astrophysics Laboratory, Org. A021S, Bldg. 252, 3251 Hanover St., Palo Alto, CA 94304, USA; aschwanden@1msal.com \\ ${ }^{2}$ National Solar Observatory, 3665 Discovery Dr., Boulder, CO 80300, USA; kreardon@nso.edu \\ ${ }^{3}$ INAF-Osservatorio Astrofisico di Arcetri, Large Enrico Fermi 5, I-50125 Firenze, Italy \\ ${ }^{4}$ Astrophysics Research Centre, School of Mathematics and Physics, Queen’s University, Belfast BT7 1NN, UK; d.jess@qub.ac.uk \\ Received 2016 February 5; revised 2016 May 4; accepted 2016 May 10; published 2016 July 21
}

\begin{abstract}
The aim of this study is to explore the suitability of chromospheric images for magnetic modeling of active regions. We use high-resolution images $\left(\approx 00^{\prime \prime} 2-0{ }^{\prime \prime} 3\right)$, from the Interferometric Bidimensional Spectrometer in the Ca II $8542 \AA$ line, the Rapid Oscillations in the Solar Atmosphere instrument in the $\mathrm{H} \alpha 6563 \AA$ line, the Interface Region Imaging Spectrograph in the $2796 \AA$ line, and compare non-potential magnetic field models obtained from those chromospheric images with those obtained from images of the Atmospheric Imaging Assembly in coronal $(171 \AA$, etc.) and in chromospheric $(304 \AA)$ wavelengths. Curvi-linear structures are automatically traced in those images with the OCCULT-2 code, to which we forward-fitted magnetic field lines computed with the Verticalcurrent Approximation Nonlinear Force Free Field code. We find that the chromospheric images: (1) reveal crisp curvi-linear structures (fibrils, loop segments, spicules) that are extremely well-suited for constraining magnetic modeling; (2) that these curvi-linear structures are field-aligned with the best-fit solution by a median misalignment angle of $\mu_{2} \approx 4^{\circ}-7^{\circ}$; (3) the free energy computed from coronal data may underestimate that obtained from cromospheric data by a factor of $\approx 2-4,(4)$ the height range of chromospheric features is confined to $h \lesssim 4000 \mathrm{~km}$, while coronal features are detected up to $h=35,000 \mathrm{~km}$; and (5) the plasma- $\beta$ parameter is $\beta \approx 10^{-5}-10^{-1}$ for all traced features. We conclude that chromospheric images reveal important magnetic structures that are complementary to coronal images and need to be included in comprehensive magnetic field models, something that is currently not accomodated in standard NLFFF codes.
\end{abstract}

Key words: Sun: chromosphere - Sun: magnetic fields - Sun: UV radiation

\section{INTRODUCTION}

While traditional methods compute the magnetic field in the solar corona by potential-field extrapolation of the photospheric line of sight (LOS) component, or by force-free extrapolation of the photospheric 3D vector field, inconsistencies have been noticed with the observed geometry of coronal loops (DeRosa et al. 2009; Sandman et al. 2009), which are supposed to trace out the magnetic field in a low plasma- $\beta$ corona. Misalignment angles between theoretical nonlinear force-free field (NLFFF) solutions and observed loop directions amount to $\mu \approx 24^{\circ}-44^{\circ}$ (DeRosa et al. 2009). Several studies have been carried out to pin down the uncertainties of NLFFF codes, regarding insufficient field of views (FOVs), the influence of the spatial resolution, insufficient constraints at the computation box boundaries, and the violation of the force-free assumption in the lower chromosphere (Metcalf et al. 2008; DeRosa et al. 2009, 2015). The latter two problems involve the knowledge of the magnetic field in the chromosphere, which represents a "missing link" between the photosphere and corona. The exploration of this missing link as a magnetic interface between photospheric magnetograms and coronal loops is the main goal of this study, where we employ chromospheric high-resolution images observed with the Interface Region Imaging Spectrograph (IRIS) (De Pontieu et al. 2014), the Interferometric Bidimensional Spectrometer (IBIS) (Cavallini 2006; Cauzzi et al. 2008; Reardon \& Cavallini 2008; Righini et al. 2010; Reardon et al. 2011), and the Rapid Oscillations in the Solar Atmosphere (ROSA) instrument (Jess et al. 2010).

What structure and manifestation has the magnetic field in the chromosphere? In this paper we define the chromosphere as the cool part of the solar atmosphere above the convection zone with a temperature range of $T_{e} \approx 5 \times 10^{3}-10^{5} \mathrm{~K}$ that extends from photospheric heights $h_{\text {phot }}=0 \mathrm{~km}$ (at $\tau_{\lambda=5000 A}$ ) upward to $h_{\text {chrom }} \approx 2000 \mathrm{~km}$ according to hydrostatic models (e.g., Vernazza et al. 1981), or up to $h_{\text {chrom }} \approx 5000 \mathrm{~km}$ according to observed dynamic phenomena (Ewell et al. 1993; Aschwanden et al. 2002). While the base of the chromosphere is dominated by the thermal pressure (with a high plasma- $\beta$, i.e., $\beta=p_{\text {th }} / p_{\text {mag }}>1$ ), a cross-over to a magnetically dominated transition region (with a low plasma- $\beta$, i.e., $\beta<1$ ) is believed to take place at the so-called "canopy height" (Gabriel 1976), which is located in an altitude range of $h \approx 0-1500 \mathrm{~km}$, depending on the location in the Quiet Sun or in sunspot regions (Buehler et al. 2015). In coronal heights above, loops are expected to be aligned with the magnetic field. Thus we expect to see the footpoints of field-aligned coronal loops above the canopy height. In addition we may often see some field-aligned dynamic structures, such as fibrils in active regions, spicules when observed above the limb, or mottles when observed in the Quiet Sun (De Pontieu et al. 2007; Pietarila et al. 2009). While there is extensive literature about the various chromospheric phenomena, we focus here on their magnetic properties only.

From measurements of the 3D vector magnetic field using the Na I $5896 \AA$ line with the Stokes Polarimeter at Mees Solar Observatory, it was found that the magnetic field is not forcefree in the photosphere, but becomes force-free at an altitude of $h \approx 400 \mathrm{~km}$ in active regions (Metcalf et al. 1995). However, a lot of chromospheric structures in weak-field regions are magnetically closed inside the chromosphere (below the canopy height), so that there is not always a link between the 
photospheric network and magnetic loops in the corona (Jendersie \& Peter 2006). The non-potentiality of the magnetic field in the chromosphere has been measured with LOS magnetograms at the National Solar Observatory's Kitt Peak Observatory (NSO/KP) using the $8542 \AA$ line, which is primarily sensitive to the magnetic field at a height of $h \approx 800 \mathrm{~km}$ (Harvey et al. 1999; Choudhary et al. 2001; de la Cruz Rodriguez et al. 2012). 3D numerical MHD simulations with the Bifrost code (Gudiksen et al. 2011) allow us to localize the contribution heights of the Ca II $8542 \AA$ line in a range of $h \lesssim 1500 \mathrm{~km}$ for the core of the line profile (Vernazza et al. 1981), confirmed also in 3D Bifrost models (Leenaarts et al. 2009). The $8542 \AA$ line is particularly suited to observe the fine structure of fibril-like features (Pietarila et al. 2009), to measure their geometry and orientation, and to determine their magnetic field-alignment and non-potentiality (Jing et al. 2011). The field-alignment of chromospheric fibrils was tested by comparing CRisp Imaging Spectro-polarimeter data (CRISP, Scharmer 2006) with Spectro-polarimeter for INfrared and Optical Regions data (SPINOR, Socas-Navarro et al. 2006), and it was found that fibrils are often oriented along the magnetic field, but not always (de la Cruz Rodriguez \& Socas-Navarro 2011). Also in MHD simulations it was found that some modeled fibrils are not field-aligned in that simulated volume (Leenaarts et al. 2015). In fact, Leenaarts et al. (2015) found that the horizontal component of the magnetic field aligns well with the plane-of-the sky direction of the observed fibrils, but the vertical component of the magnetic field is not necessarily aligned with the fibrils. IBIS observations from the Dunn Solar Telescope (DST) in New Mexico found that fibrils are aligned with the magnetic field with an uncertainty of $\mu \lesssim 10^{\circ}$ (Schad et al. 2013). These IBIS observations were done with the $\mathrm{H}_{\mathrm{I}} 10830 \AA$ line, which typically forms somewhat higher in the chromosphere than the 8542 A line.

In this study we conduct non-potential field modeling of chromospheric structures and coronal loops with a particular NLFFF code that is based on a vertical-current approximation (VCA), and we perform automated tracing of coronal loops and chromospheric curvi-linear features. A short description of the VCA-NLFFF code is provided in Section 2, while a more complete account is given in Aschwanden (2016). Data analysis of Atmospheric Imager Assembly (AIA) (Lemen et al. 2012) and Helioseismic Magnetic Imager (HMI) (Scherrer et al. 2012) data from the Solar Dynamics Observatory (SDO) (Pesnell et al. 2011), from the (IRIS) (De Pontieu et al. 2014), from the (IBIS) (Cavallini 2006), and from the (ROSA) (Jess et al. 2010) instrument are presented in Section 3, and discussion and conclusions are offered in Sections 4 and 5.

\section{METHOD}

Traditional NLFFF codes use the 3D vector field $\boldsymbol{B}(x, y)=\left[B_{x}(x, y), B_{y}(x, y), B_{z}(x, y)\right]$ from a vector magnetograph instrument as input for the photospheric boundary (in the $(x, y)$-plane), and use a height-extrapolation scheme to compute magnetic field lines that match the boundary condition, the divergence-freeness, and the forcefreeness conditions. Examples and comparisons of such recent NLFFF codes are given in Metcalf et al. (2008) and DeRosa et al. (2009, 2015), including the optimization method (Wheatland et al. 2000; Wiegelmann \& Inhester 2010), the magneto-frictional method (Valori et al. 2007, 2010), the
Grad-Rubin method (Amari et al. 2006; Wheatland 2007), and others.

Here we use an alternative method which is called the Vertical Current Approximation Nonlinear Force-free Field (VCA-NLFFF) method. The theoretical model assumes a variable amount of vertical currents associated with each magnetic field concentration, which introduces a helical twist about the vertical axis (Figure 1). A detailed description and performance tests of measuring the non-potential magnetic energy is documented in Aschwanden (2016). The VCANLFFF code consists of three tasks: (1) the decomposition of a LOS magnetogram $B_{z}(x, y)$ into a finite number of (subphotospheric) buried unipolar magnetic charges, from which the potential field is calculated by superimposition of the potential fields $B(r) \propto r^{-2}$ of each magnetic charge; (2) an automated loop tracing code extracts the 2D-coordinates $[x(s), y(s)]$ of coronal loops or chromospheric fibrils (as a function of the loop length coordinate $s$ ) from EUV, soft X-ray, or $\mathrm{H} \alpha$ images at various wavelenghts; and (3) a parameterized NLFFF model that contains the potential-field parameters of each magnetic charge plus a non-potential field component in terms of vertical currents above each magnetic charge is then forward-fitted to the automatically traced loop coordinates $[x(s), y(s)]$ by minimization of the relative $3 \mathrm{D}$ misalignment angles between the theoretical non-potential field model and the observed 2D loop coordinates. Implicitly, the assumption is made that the traced loops and fibrils are located in a low plasma- $\beta$ environment, as it is the case in most coronal and upper chromospheric regions. This code uses only the LOS magnetogram $B_{z}(x, y)$ to constrain the potential field, while the transverse photospheric components $B_{x}(x, y)$ and $B_{y}(x, y)$ are not used in the reconstruction of the non-potential field model, since the loop coordinates yield sufficient constraints. The unknown height information $z(s)$ of the traced 2Dprojected loops and fibrils, with the coordinates $[x(s), y(s)]$, is obtained from the best fit of a forward-fitted geometric 3D model, where each loop segment is parameterized by a circular segment in the loop plane with 4 free parameters (i.e., minimum and maximum height of loop segment $h_{\min }$ and $h_{\text {max }}$, and two azimuth angles $\phi_{1}$ and $\phi_{2}$ at the start and the end of the circular segment, see visualization in Figure 11 of Aschwanden 2016). In the forward-fitting procedure that leads to a best-fit VCA-NLFFF solution, it is implicitly assumed that the loops and fibrils follow the magnetic field. The chief advantages of the VCA-NLFFF code over traditional NLFFF codes are the circumvention of the unrealistic assumption of a force-free photosphere in the magnetic field extrapolation method, the capability to minimize the misalignment angles between observed coronal loops (or chromospheric fibril structures) and theoretical model field lines, as well as computational speed. The fitted VCA-NLFFF solution is entirely constrained by the observed loop shapes inside the computation box, and thus does not suffer from assumptions of the field model at the boundaries of the computation box, in contrast to standard NLFFF codes. The theory of the VCA is originally derived in Aschwanden (2013a), while the numerical VCA-NLFFF code has been continuously developed and improved in a number of previous studies (Aschwanden et al. 2008, 2012, 2013, 2014b, 2014a; Aschwanden 2010, 2013b, 2013c, 2015; Aschwanden \& Sandman 2010; Sandman \& Aschwanden 2011; Aschwanden \& Malanushenko 2013). 

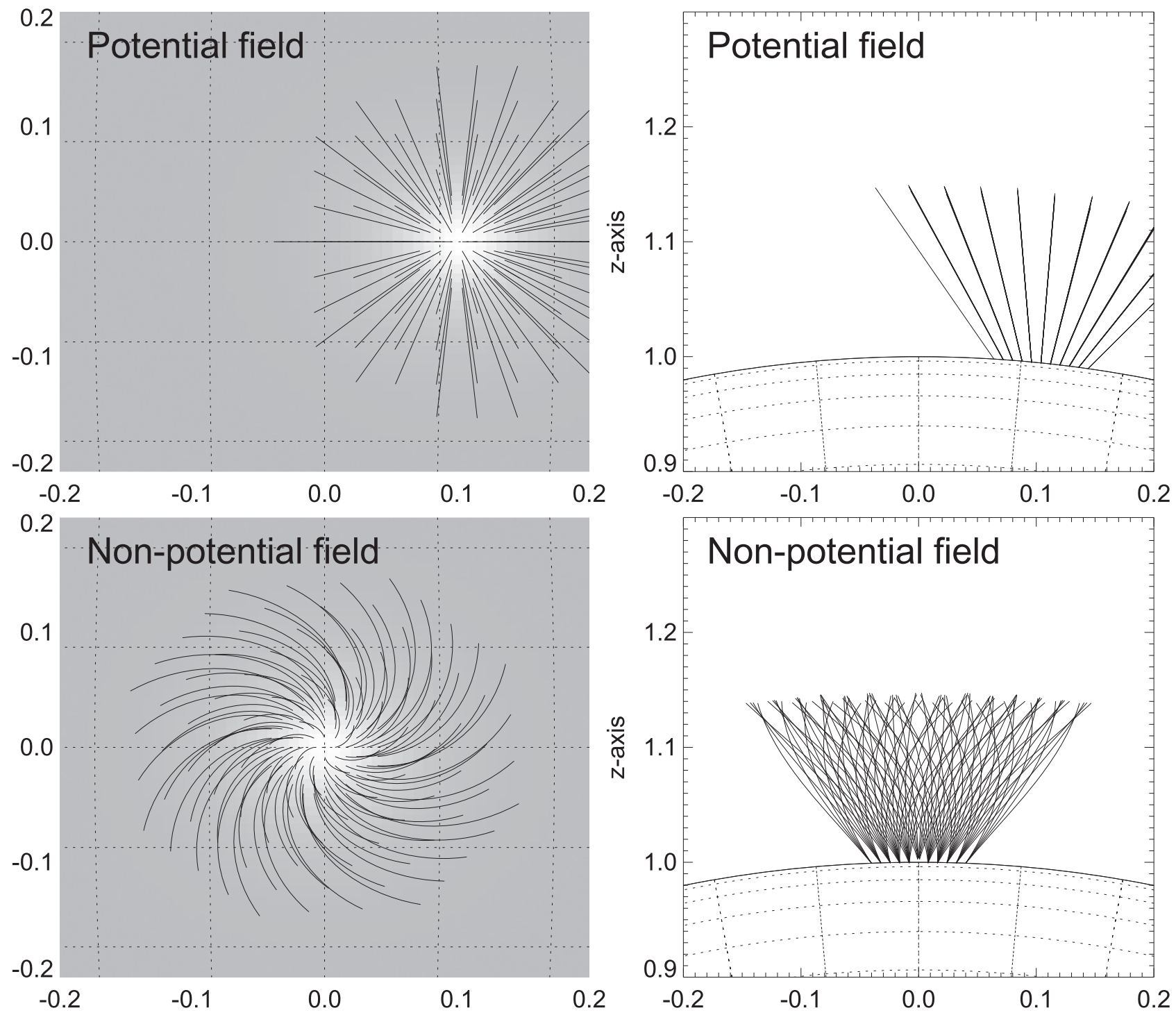

Figure 1. Magnetic field lines of a single unipolar magnetic charge, mimicking a single sunspot, computed for a potential field (top panels) and for a non-potential field based on the vertical-current approximation (VCA-NLFFF code), which introduces a helical twist about the vertical axis (bottom panels). The left panels depict a projection from top down to the solar surface, while the right panels show a side view. The grayscale indicates the corresponding line of sight magnetogram.

The only other existing code that obtains a NLFFF solution by forward-fitting to coronal loops has been developed by Malanushenko et al. (2009, 2011, 2012, 2014), implemented by a quasi-Grad-Rubin method.

\section{OBSERVATIONS AND DATA ANALYSIS}

Since the motivation of this study is the exploration of chromospheric structures regarding their suitability for magnetic field modeling, we choose observations with the highest available spatial resolution that show crisp chromospheric structures. We found such suitable data from the IBIS instrument in the $\mathrm{Ca}$ II 8542 line, and from both the IBIS and ROSA instrument in the $6563 \mathrm{H} \alpha$ line, both having a spatial resolution of $\approx 0, " 2-00^{\prime \prime} 3$. We analyze such data from three different observing runs (Table 2) at the NSO/SP DST (2010 August 3; 2014 August 24, 2014 August 30), and complement these observations with $I R I S$, AIA/SDO, and $\mathrm{HMI} / S D O$ data.

\subsection{Observations of 2010 August 3}

The IBIS, a ground-based dual Fabry-Perot interferometer, records images in the wavelength range of $5400-8600 \AA$ (Cavallini 2006; Reardon \& Cavallini 2008; Righini et al. 2010; Reardon et al. 2011), is installed on the DST at the NSO/SP facility, has a FOV of $95^{\prime \prime}$, a pixel size of $0 . \prime 0976$, a diffraction-limited resolution of $1.22 \lambda / D=$ $0 . " 14 /$ pixel $=0$. .' 28 at $8542 \AA$, and acquired images for active region NOAA 11092 on 2010 August 3, 15:03-15:43 UT. We analyze a $240^{\prime \prime} \times 240^{\prime \prime}$ mosaic image taken in the wavelength of Ca II $8542 \AA$ and H-alpha $6563 \AA$ (described also in Jing et al. 2011; Reardon et al. 2011), but we limit the FOV to a square with a size of FOV $=0.10 R_{\odot}$ centered on the primary sunspot of NOAA 11092, covering the ranges of $x=[-0.0159,0.0841] R_{\odot}$ in EW direction and $y=[0.0608$, $0.1608] R_{\odot}$ in NS direction, centered at a heliographic position of N12W02, almost near the center of the solar disk. Since IBIS images cover a limited portion of the solar disk only, the 


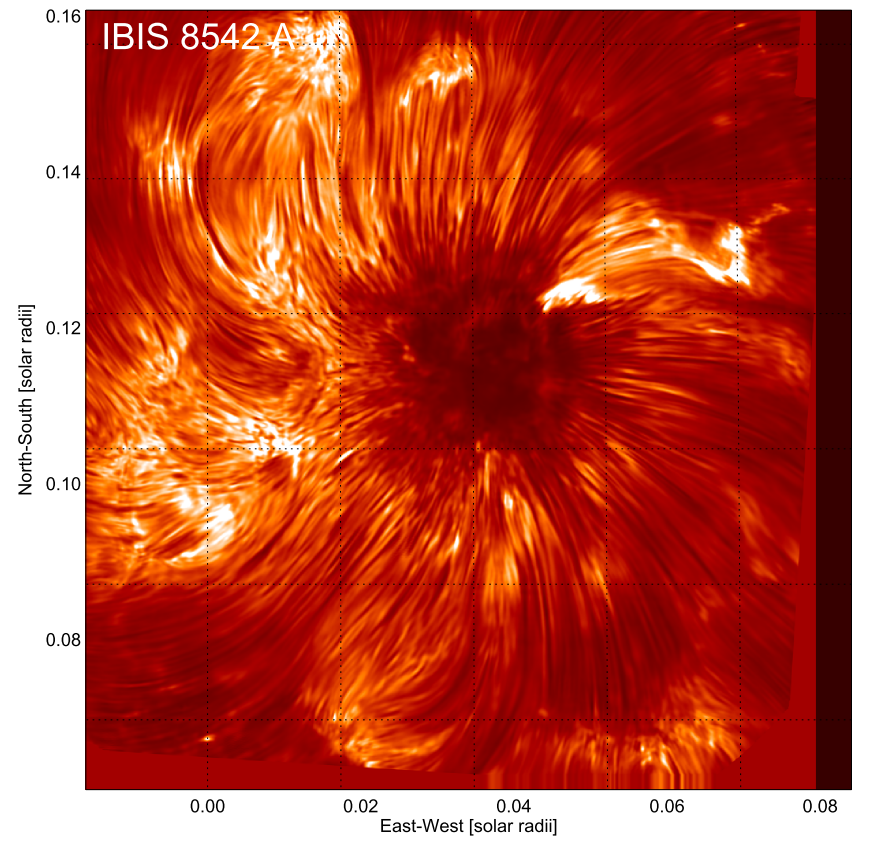

Figure 2. IBIS image of active region NOAA 11092 observed on 2010 August 3, 15:03-15:43 UT in the Ca II $8542 \AA$ line. The displayed field of view captures a subimage centered at the sunspot with a width of FOV $=0.10 R_{\odot}$ and a spatial pixel size of $0 . \prime 28$.

coalignment with full-disk images was carried out with an AIA $1600 \AA$ image at the mid-time of the IBIS image acquisition time interval, at 15:23:00 UT. A rendering of the analyzed subimage of IBIS $8542 \AA$ is shown in Figure 2, where the umbra of the sunspot appears dark, surrounded by a wreath of fibrils that spiral in curved trajectories from the penumbra away from the sunspot, similar to the non-potential field model (based on vertical currents) shown in Figure 1 (bottom left panel, on arbitrary scale), though with opposite chirality.

A highpass-filtered version of the original IBIS 8542 image (Figure 2) is shown in Figure 3 (top panel), which is produced by subtracting a finestructure image (the original image smoothed with a boxcar of $n s m_{1}=3$ pixels) from a lowpassfiltered image (the original image smoothed with a boxcar of $n s m_{2}=n s m_{1}+2=5$ pixels). This filter enhances structures with a width of $w \approx 3$ pixels $\approx 0$ ! $3 \approx 200 \mathrm{~km}$. We perform an automated loop tracing run with the OCCULT-2 code (Aschwanden et al. 2013) setting the maximum number of analyzed structures to $n_{\text {struc }}=10,000$, while all other control parameters are set to standard values (Table 1). This run identifies a total of 1193 curvi-linear structures (Figure 3, bottom panel). Note the absence of curvi-linear fine structure in the sunspot umbra, while fibrils or loop segments are filling most of the penumbral area and surrounding outskirts of the active region. The richness of curvi-linear structures makes the image highly suitable for magnetic field modeling and quantitative tests of field-alignment. For a comparison of the sensitivity and efficiency of the automated feature detection algorithm OCCULT-2, see also Figure 2 in Jing et al. (2011), where the same image has been processed with a union-finding segmentation algorithm (Sedgewick 2002).

In order to complement the chromospheric image of IBIS with coronal images, we include AIA/SDO images, at coronal wavelengths of $94,131,171,193,211,335 \AA$, as well as $304 \AA$ at cooler chromospheric temperatures (see temperature ranges in Table 2). AIA subimages with the same FOV as the IBIS

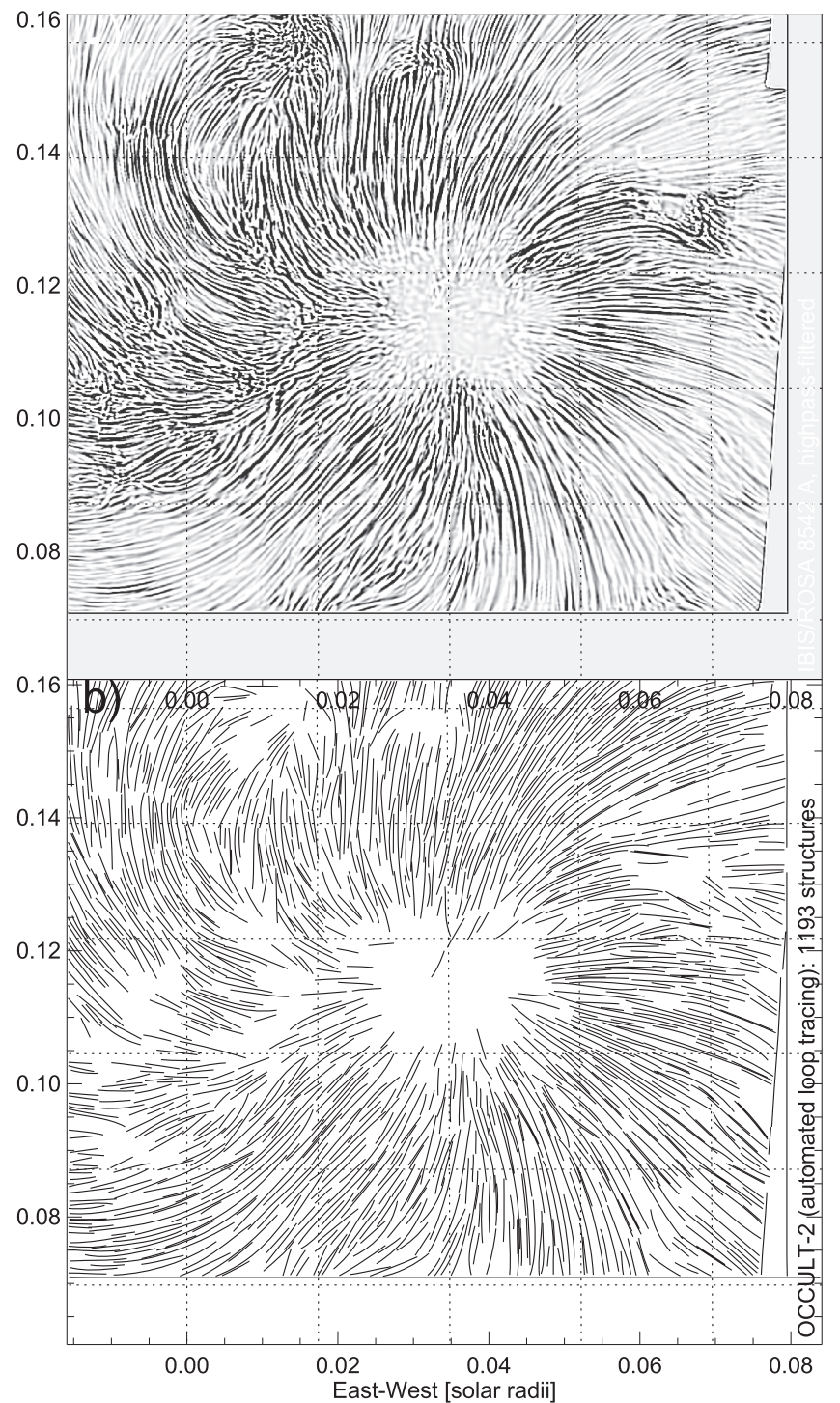

Figure 3. A highpass-filtered version of the original IBIS $8542 \AA$ subimage shown in Figure 2 (top panel), along with 1193 automatically traced curvilinear structures using the OCCULT-2 code (bottom panel), which may consist of chromospheric fibrils or footpoints of coronal loops.

image (Figures 2-3) are shown in Figure 4 (left column), with highpass-filtered versions (Figure 4, middle column), and automated loop tracings (Figure 4, right column). While the original images show fans that contain bundles of loops rooted in the sunspot, the highpass-filtered images reveal loops with narrower widths, which are detected and localized with the OCCULT-2 code. It appears that the radially extending coronal loops seen in AIA have a similar orientation as the chromospheric structures seen in the IBIS image. However, since IBIS has a six time higher spatial resolution than AIA on a 1D linear scale (corresponding to 36 times more details in a 2D area), the detected loop features are much less numerous in the AIA images $(\approx 15-40$ per wavelength in Figure 4$)$ than in the IBIS image $\left(n_{\mathrm{IBIS}} \approx 1200\right.$ in Figure 3$)$. This is mostly a consequence of the much higher spatial resolution of IBIS. The 36 times larger amount of image area information in IBIS let us expect about $n_{\text {AIA,pred }} \approx 1200 / 36=33$ loop structures per AIA image, which is indeed the case, according to the counts of 
Table 1

Data Selection Parameters and Adjustable Control Parameters of the VCA-NLFFF Forward-fitting Code Used in this Study

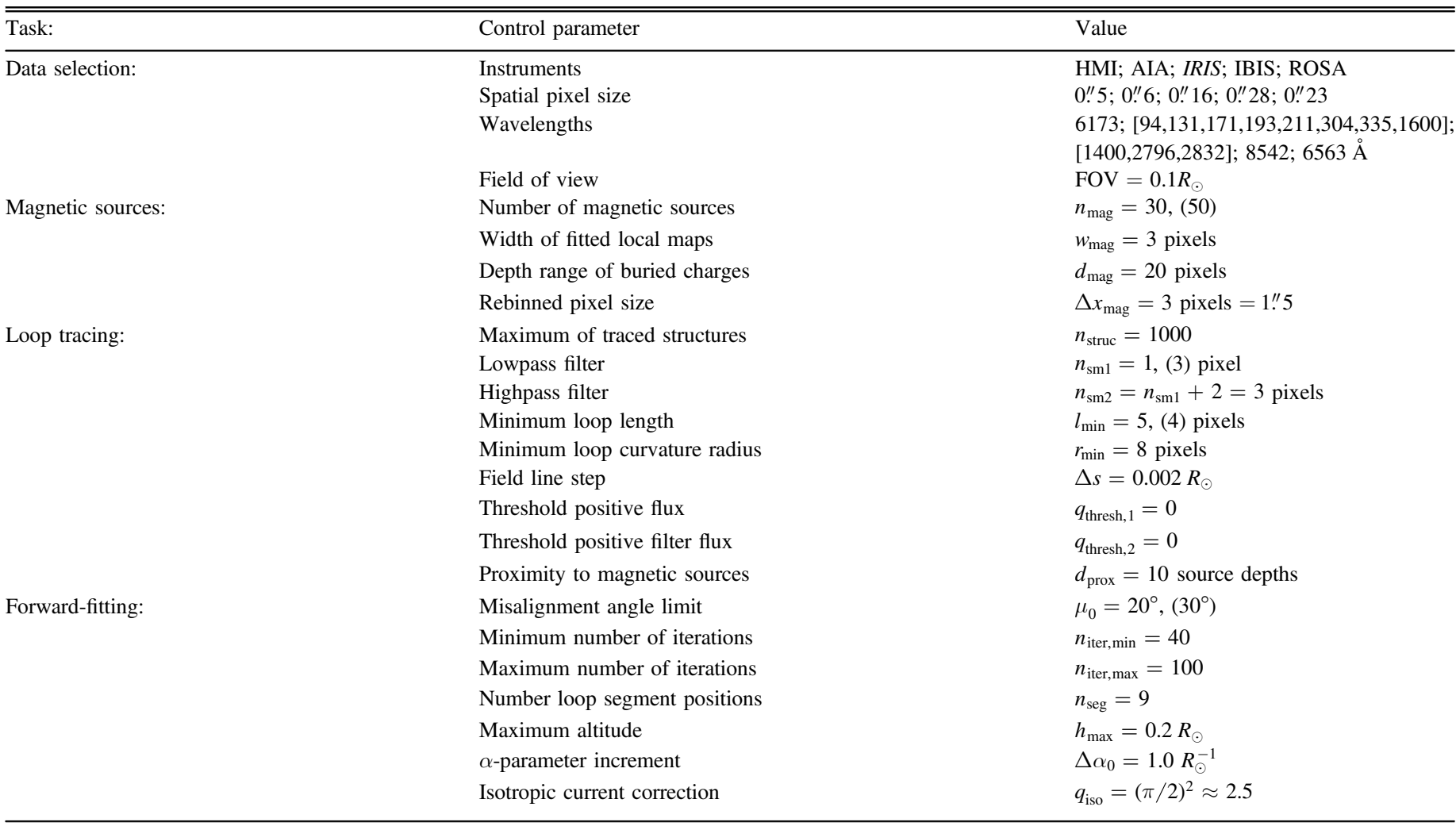

Table 2

Observation Date and Times, Active Region Numbers, Instruments, and Wavelength Ranges

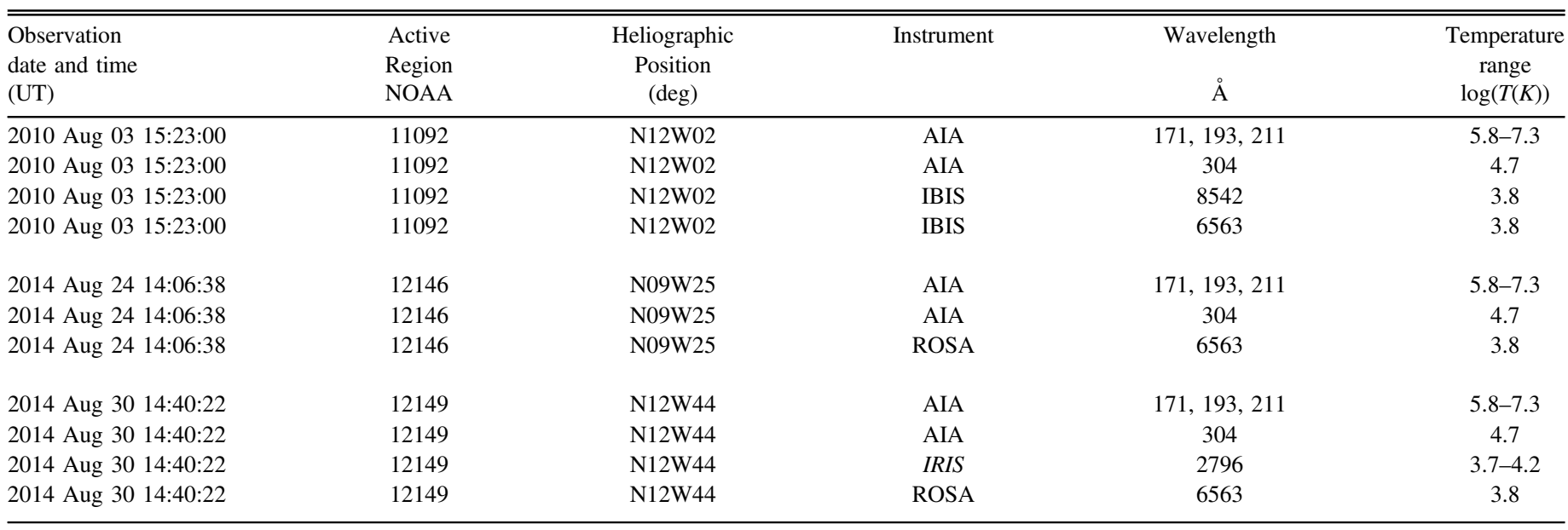

$n_{\mathrm{AIA}} \approx 15-40$ in Figure 4 . Alternatively, the corona may be inherently less fine-structured than the chromosphere, or may have a lower filling factor of detected loop segments. Unfortunately, no contemporaneous IRIS images were available at this time.

\subsection{Observations of 2014 August 24}

A second data set that provides $\mathrm{ROSA} \mathrm{H} \alpha$ images at $6563 \AA$ was obtained during a campaign at the NSO/SP DST on 2014 August 24, 13:55-15:56 UT. The ROSA/HARDcam has a diffraction-limited resolution of $1.22 \lambda / D=0$ ! $11 /$ pixel $=0.22$ at the $\mathrm{H} \alpha$ wavelength $6563 \AA$. We select an image obtained at 14:06:38 UT, which is also shown in Figure 2 of Jess et al. (2015). We select a subimage with a square FOV of size $\mathrm{FOV}=0.10 R \odot$ that centers on the primary sunspot of NOAA 12146 , covering the ranges of $x=[0.3674,0.4674] R_{\odot}$ in EW direction and $y=[-0.0112,0.0888] R_{\odot}$ in $\mathrm{NS}$ direction, which is centered at a heliographic position of N09W25, being about $0.4 R_{\odot}$ away from disk center. Unfortunately, no cotemporal IRIS images are available at this time either.

\subsection{Observations of 2014 August 30}

A third data set of ROSA $\mathrm{H} \alpha$ images during the same week of the the observing campaign was obtained on 2014 August 

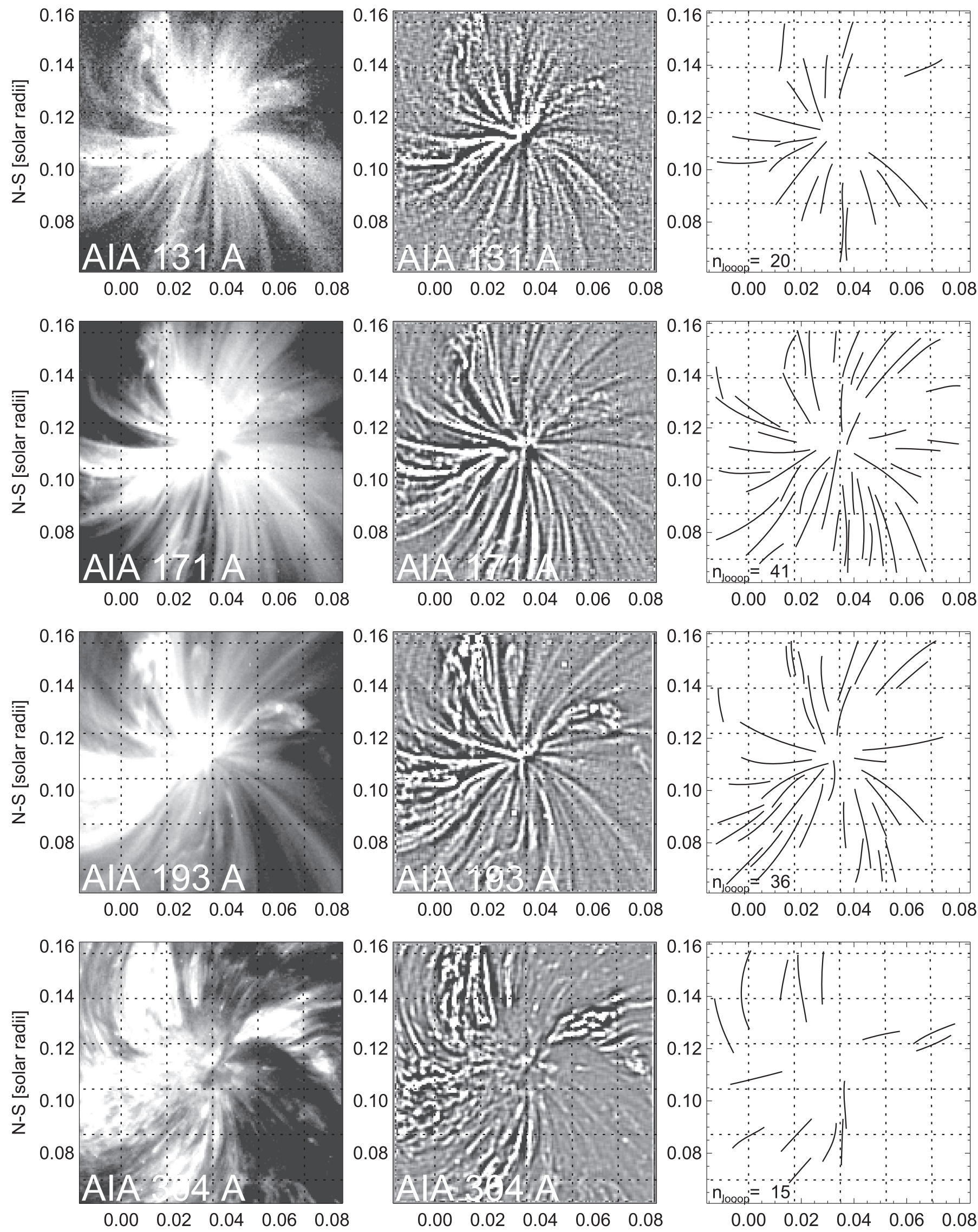

Figure 4. AIA/SDO images in the wavelengths of 131, 171, 193, and $304 \AA$ on a logarithmic scale (left column), their highpass-filtered counterparts on a linear scale and smoothed with a boxcar of $n_{\mathrm{sm} 1}=3$ pixels (middle column), and the results of automated loop tracings obtained with the OCCULT-2 code (right column). 
30, 14:37-17:46 UT. We select a subimage with a square FOV of size $\mathrm{FOV}=0.10 R_{\odot}$ that centers on the primary sunspot of NOAA 12149 , covering the ranges of $x=[0.6295,0.7295] R_{\odot}$ in EW direction and $y=[0.0375,0.1375] R_{\odot}$ in NS direction, which is centered at a heliographic position of N12W44, being about $0.7 R_{\odot}$ away from disk center. Such large distances from disk center may introduce larger errors in the deconvolution of magnetograms into unipolar magnetic charges (although our deconvolution technique takes the $3 \mathrm{D}$ effects into account), as well as be less suitable for automated tracing of curvi-linear features due to the confusion caused by a larger number of superimposed structures and a higher degree of foreshortening. Nevertheless, we include these less than ideal data in our analysis in order to test the accuracy of magnetic energy measurements with our VCA-NLFFF code.

In addition, IRIS (as well as Hinode SOT/SP) was pointing to the same active region at this time, so that we have an independent comparison of chromospheric images with ROSA. From IRIS we choose the $\mathrm{Mg}_{\text {II }} h / k$ lines at 2796 and $2802 \AA$ for our analysis, which are sensitive to a chromospheric temperature range of $\log (T)[k])=3.7-4.2$ (or 5000-16,000 K) (Carlsson \& Leenaarts 2012).

\subsection{Results of Magnetic Modeling}

We perform magnetic field modeling of the 2010 August 3, 15:23 UT data in four different wavelength groups, in the coronal lines of AIA/SDO $(94,131,171,193,211,335 \AA)$ (Figure 5), in the AIA/SDO wavelength of He II $304 \AA$ (Figure 6), in the chromospheric IBIS $8542 \AA$ image (Figure 7), and in the IBIS H $\alpha 6563 \AA$ line (Figure 8). The automatically traced loop-like structures are visualized with yellow curves in Figures 5-8, while the best-fit magnetic field lines obtained with the VCA-NLFFF code are shown in red color. The various input parameters of the VCA-NLFFF code are listed in Table 1. We summarize the key results of our magnetic modeling runs in Table 3, which includes the number of (automatically detected) field-aligned loop structures $n_{\text {det }}$, the number of loops auto-selected for fitting $n_{\text {loop }}$ (by imposing a progressive elimination of structures with large misalignment angles during the forward-fitting procedure), the median misalignment angles $\left(\mu_{2}\right)$,

$$
\mu_{2}=\cos ^{-1}\left(\frac{\left(\boldsymbol{B}^{\text {theo }} \cdot \boldsymbol{x}^{\text {obs }}\right)}{\left|\boldsymbol{B}^{\text {theo }}\right| \cdot\left|\boldsymbol{x}^{\text {obs }}\right|}\right)
$$

where $\boldsymbol{B}$ represents the 3D magnetic field vector of the theoretical field model $\boldsymbol{B}^{\text {theo }}$, while $\boldsymbol{x}^{\text {obs }}$ is the observed 2Dvector direction tangent to an observed loop tracing, while the 2D misalignment angle is measured from the projected 2D vectors without the LOS component, i.e., $\left.\boldsymbol{B}=\left(B_{x}, B_{y}, 0\right)\right]$. In analogy, we define also a 3D misalignment angle $\mu_{3}$, where the third component of $\boldsymbol{x}(x, y, z)$ is taken into account from the best-fit geometric 3D model of circular segments. In Table 3 we further include the total potential magnetic field energies $\left(E_{\mathrm{P}}\right)$,

$$
E_{\mathrm{P}}=\int \frac{1}{8 \pi} B_{\mathrm{P}}(\boldsymbol{x})^{2} d V
$$

which together with the non-potential energies, $\left(E_{\mathrm{NP}}\right)$,

$$
E_{\mathrm{NP}}=\int \frac{1}{8 \pi} B_{\mathrm{NP}}(\boldsymbol{x})^{2} d V,
$$

quantifies the free energy ratio,

$$
q_{\text {free }}=E_{\mathrm{NP}} / E_{\mathrm{P}}-1 .
$$

In order to estimate the uncertainty of the results, each of the 11 forward-fits listed in Table 3 has been repeated five times with different input parameters (minimum loop segment length $l_{\min }=4,5$; number of unipolar magnetic sources $n_{\text {mag }}=30,50$, smoothing boxcar length $n_{\mathrm{sm} 1}=1,3$, upper limit of fitted misalignment angles $\mu_{0}=20^{\circ}, 30^{\circ}$ ), and the mean and standard deviations are listed in Table 3.

For coronal wavelengths (AIA 94, 131, 171, 193, 211, $335 \AA$ ) we find a large number of field-aligned structures, in the range of $n_{\text {det }} \approx 200$ (Table 3 ), which is expected since coronal loops are supposed to follow the magnetic field. A new result is that we find a large number of field-aligned structures (fibrils) in the IBIS Ca II $8542 \AA$ line $\left(n_{\mathrm{det}} \approx 700\right.$; Figure 7$)$, in the IBIS $\mathrm{H} \alpha 6563 \AA$ line $\left(n_{\mathrm{det}} \approx 700\right.$; Figure 8$)$, and in the IRIS Mg II $2796 \AA$ line $\left(n_{\mathrm{det}} \approx 200\right.$, Table 3$)$. The accuracy of the field alignment is measured with the 2D misalignment angle $\mu_{2}$, amounting to $\mu_{2} \approx 4^{\circ}-5^{\circ}$ for IBIS (Figures 7 and 8 ). The number of field-aligned loops is lowest for the AIA $304 \AA$ wavelength, in the range of $n_{\mathrm{det}} \approx 50$ (Table 3 ), produced by the chromospheric He II line with the highest temperature sensitivity at $\log (T[K])=4.7$ or $T \approx 50,000 \mathrm{~K})$. The scarce number of detected curvi-linear features most likely results from confusion problems of the OCCULT-2 code in the detection of overlaying chromospheric and coronal structures. Alternatively, a higher opacity of the line formation process could play a role.

A fundamental limitation is the 2D nature of the observations, which applies to the observed EUV images as well as to the automatically traced curvi-linear features, while our forward-fitted magnetic field (VCA-NLFFF) code yields a 3D model. Since the LOS coordinate $z$ cannot be measured, we can observe the 2D misalignment angles $\mu_{2}$ only, which are measured in the plane-of-sky (without LOS component). The relationship between the two angles is expected to be $\mu_{2}=\sqrt{2 / 3} \mu_{3} \approx 0.8 \mu_{3}$ for isotropic errors. The measured median values have a ratio of $\mu_{2} / \mu_{3} \approx 0.6$ (Table 3 ), where $\mu_{2}$ is measured from the 2D-projected loop angles, while $\mu_{3}$ (Equation (1)) is inferred from the fits of the 3D-model field lines. This indicates that the altitude errors (along the LOS) in the magnetic field models are larger than the horizontal errors. Nevertheless, it is gratifying to see that, through the use of high-resolution images, the errors of the theoretical (verticalcurrent) magnetic field model combined with the errors of automated curvi-linear tracing could be beaten down to an unprecedented low value of $\mu_{2}=4^{\circ}-5^{\circ}$, compared with the discrepancy of $\approx 24^{\circ}-44^{\circ}$ found between traditional NLFFF models and observed loops (DeRosa et al. 2009; Sandman et al. 2009). There is no other NLFFF model known that matches the loop directions with such a high degree of accuracy.

The most interesting parameter is the free energy ratio in active regions. The most accurate measurements of the free energy were achieved for the first case (NOAA 11092 observed on 2010 August 3), where the coronal AIA data yield a free energy ratio of $q_{\text {free }}=3 \% \pm 1 \%$, while the IBIS Ca II $8542 \AA$ 
20100803_152300, time step=1, AIA/SDO 94, 131, 171, 193, 211, 335 A
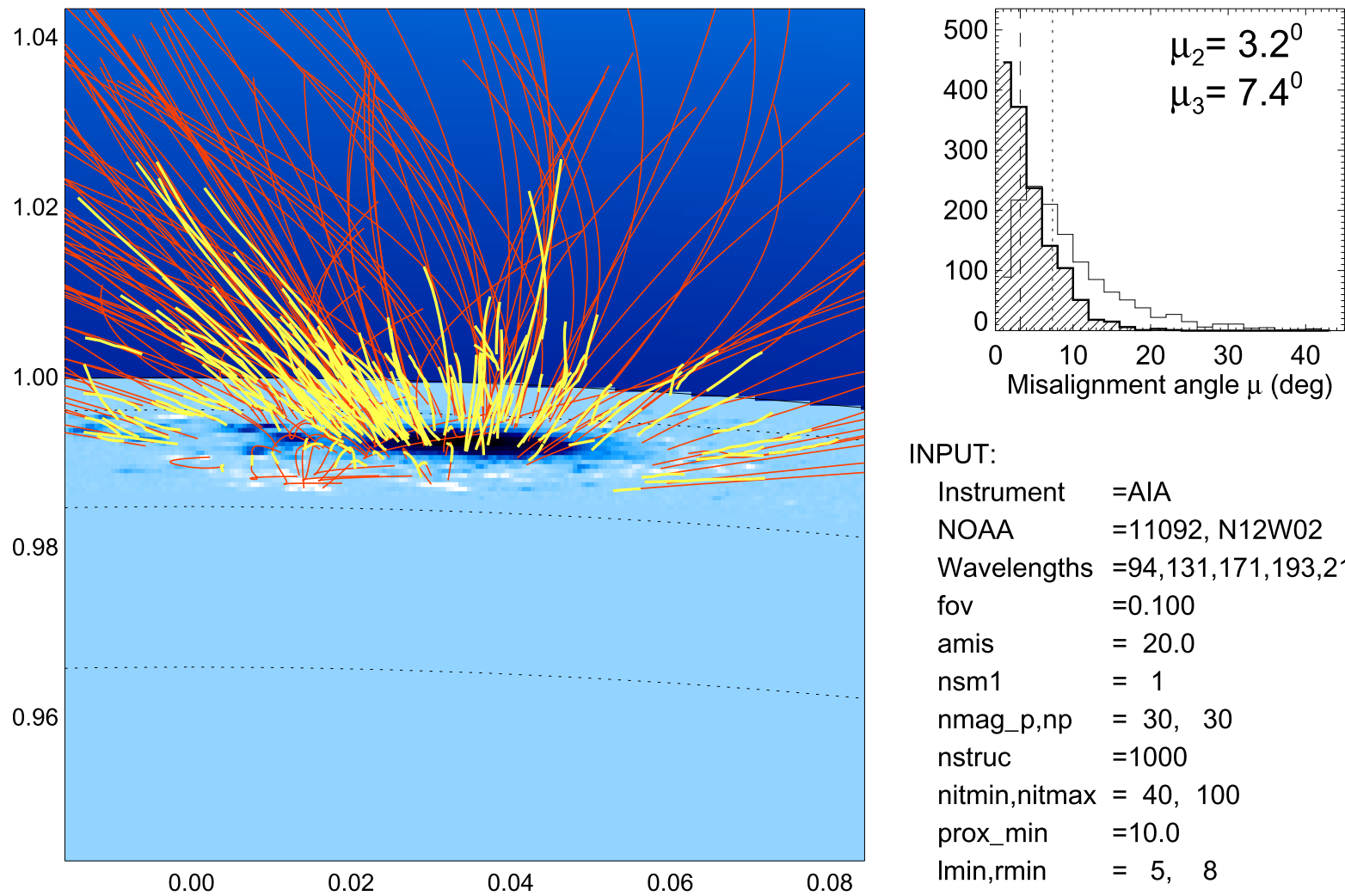

INPUT:

$\begin{array}{ll}\text { Instrument } & =\text { AIA } \\ \text { NOAA } & =11092, N 12 \text { W02 } \\ \text { Wavelengths } & =94,131,171,193,211,335 \mathrm{~A} \\ \text { fov } & =0.100 \\ \text { amis } & =20.0 \\ \text { nsm1 } & =1 \\ \text { nmag_p,np } & =30, \quad 30 \\ \text { nstruc } & =1000 \\ \text { nitmin,nitmax } & =40, \quad 100 \\ \text { prox_min } & =10.0 \\ \text { Imin,rmin } & =5, \quad 8 \\ \text { qthresh1,2 } & =0.00,0.00\end{array}$

OUTPUT:

$[\mathrm{x} 1, \mathrm{x} 2] \quad=-0.0159,0.0841$

[y1,y2] $\quad=0.0608,0.1608$

dpix HMI $=0.0005,0.51$ arcsec

dpix AIA $=0.0006,0.61 \mathrm{arcsec}$

nloop,ndet $\quad=155 / 209$

nloop/ndet $=0.742$

misalign $=3.2,7.4 \mathrm{deg}$

div-free $\quad=1.2 \mathrm{e}-02$

force-free $\quad=1.9 \mathrm{e}-02$

weight curr $=9.9 \mathrm{e}-01$

qe_rebin $=0.985$

qe_model $=1.014$

qiso_corr $=2.467$

E_P $\quad=581.3 \times 10^{30} \mathrm{erg}$

E_free $\quad=16.4 \times 10^{30} \mathrm{erg}$

E_NP/E_P $=1.028$

Iterations $\quad=42$

$\mathrm{CPU} \quad=98.0 \mathrm{~s}$

Figure 5. The automated curvi-linear feature tracing in the AIA images (2010 August 03, 15:23 UT) in 6 AIA wavelengths of 94, 131, 171, 193, 211, 335 A (yellow curves) are shown, overlaid on the best-fit solutions of the magnetic field model using the VCA-NLFFF code (red curves), and the observed HMI magnetogram (blue background image), from the line of sight view in the $(x, y)$-plane (bottom panel) and the orthogonal projection in the $(x, z)$-plane (top panel). A histogram of the 2D and 3D misalignment angles and various input and output parameters are shown in the top right-hand panel. 


\section{3_152300, time step=1, AIA/SDO 304 A}
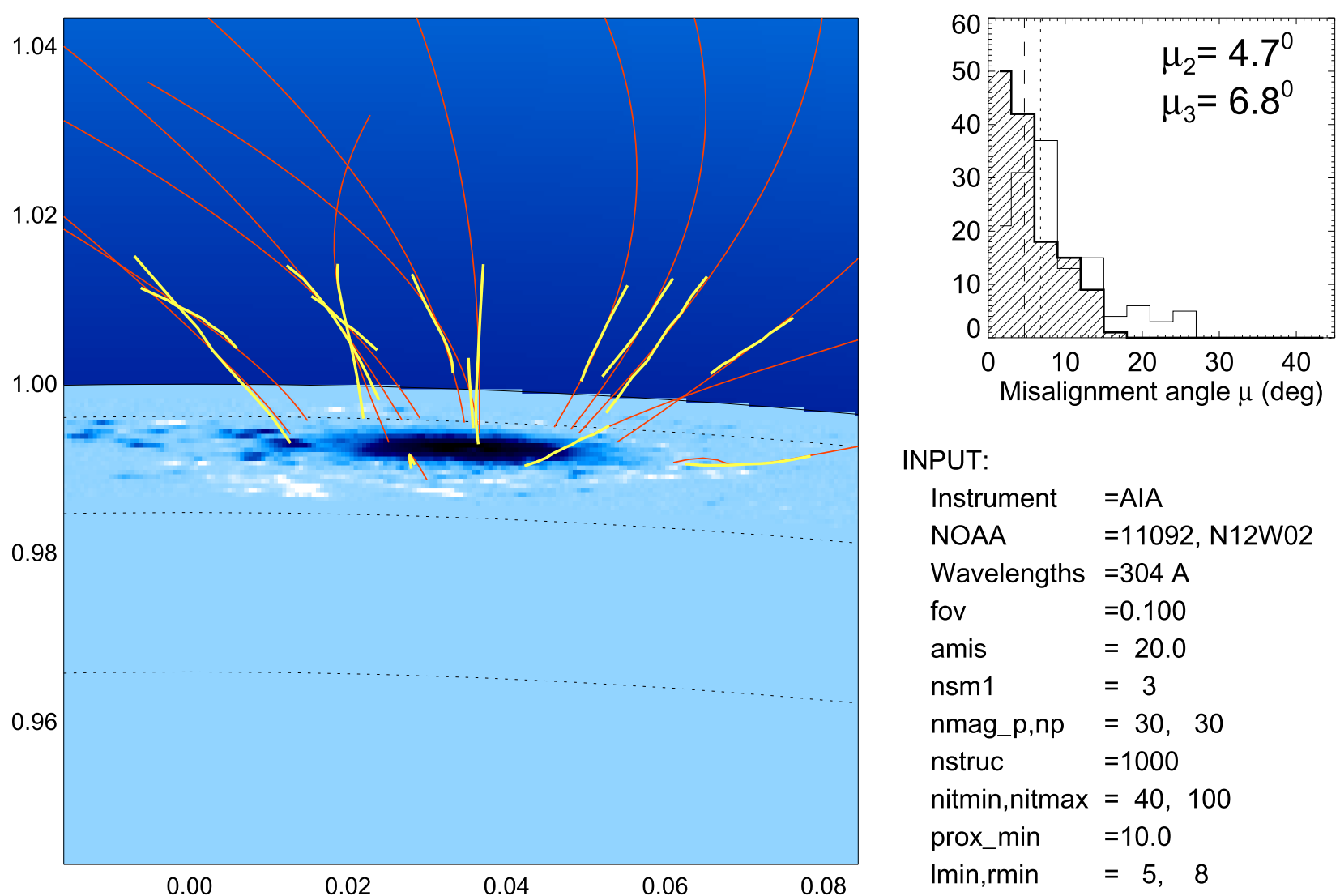

INPUT:

$\begin{array}{ll}\text { Instrument } & =\text { AIA } \\ \text { NOAA } & =11092, N 12 \mathrm{~W} 02 \\ \text { Wavelengths } & =304 \mathrm{~A} \\ \text { fov } & =0.100 \\ \text { amis } & =20.0 \\ \text { nsm1 } & =3 \\ \text { nmag_p,np } & =30,30 \\ \text { nstruc } & =1000 \\ \text { nitmin,nitmax } & =40,100 \\ \text { prox_min } & =10.0 \\ \text { Imin,rmin } & =5,8 \\ \text { qthresh1,2 } & =0.00,0.00\end{array}$

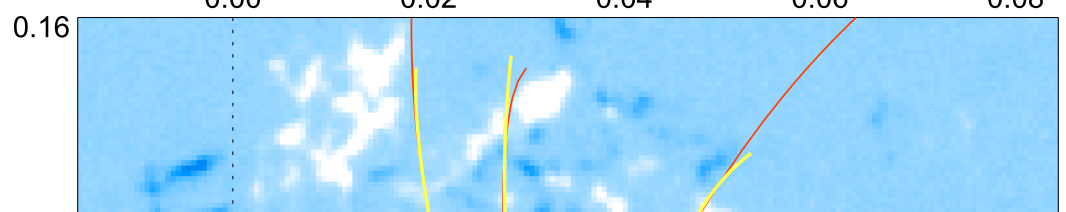

OUTPUT:

0.14

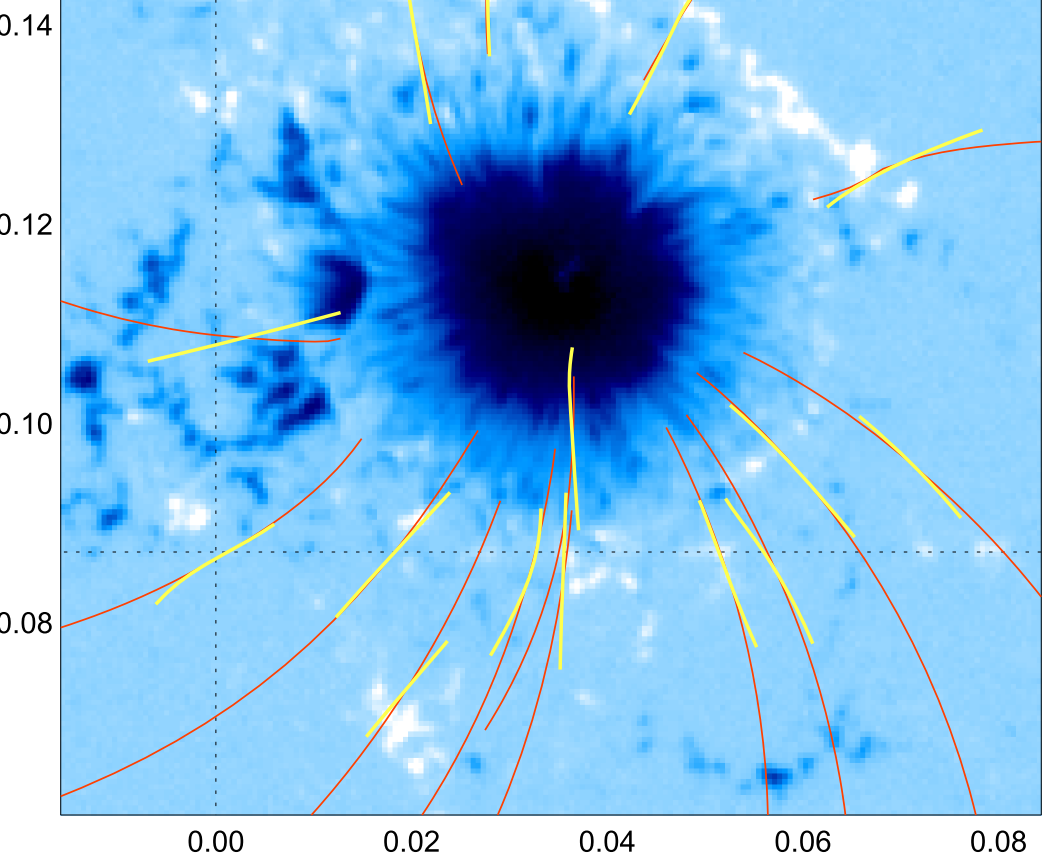

$\begin{array}{ll}\text { [x1,x2] } & =-0.0159,0.0841 \\ \text { [y1,y2] } & =0.0608,0.1608 \\ \text { dpix HMI } & =0.0005,0.51 \mathrm{arcsec} \\ \text { dpix AIA } & =0.0006,0.61 \mathrm{arcsec} \\ \text { nloop,ndet } & =15 / \quad 22 \\ \text { nloop/ndet } & =0.682 \\ \text { misalign } & =4.7, \quad 6.8 \mathrm{deg} \\ \text { div-free } & =1.2 \mathrm{e}-02 \\ \text { force-free } & =1.9 \mathrm{e}-02 \\ \text { weight curr } & =9.9 \mathrm{e}-01 \\ \text { qe_rebin } & =0.985 \\ \text { qe_model } & =1.014 \\ \text { qiso_corr } & =2.467 \\ \text { E_P } & =581.3 \times 10^{30} \mathrm{erg} \\ \text { E_free } & =24.9 \times 10^{30} \mathrm{erg} \\ \text { E_NP/E_P } & =1.043 \\ \text { Iterations } & =45 \\ \text { CPU } & =18.1 \mathrm{~s}\end{array}$

Figure 6. Automated feature tracking is applied to the AIA image (2010 August 03, 15:23 UT) in the wavelength of H I $304 \AA$ wavelength, otherwise similar representation as in Figure 5. 
20100803_152300, time step=1, IBIS 8542 A
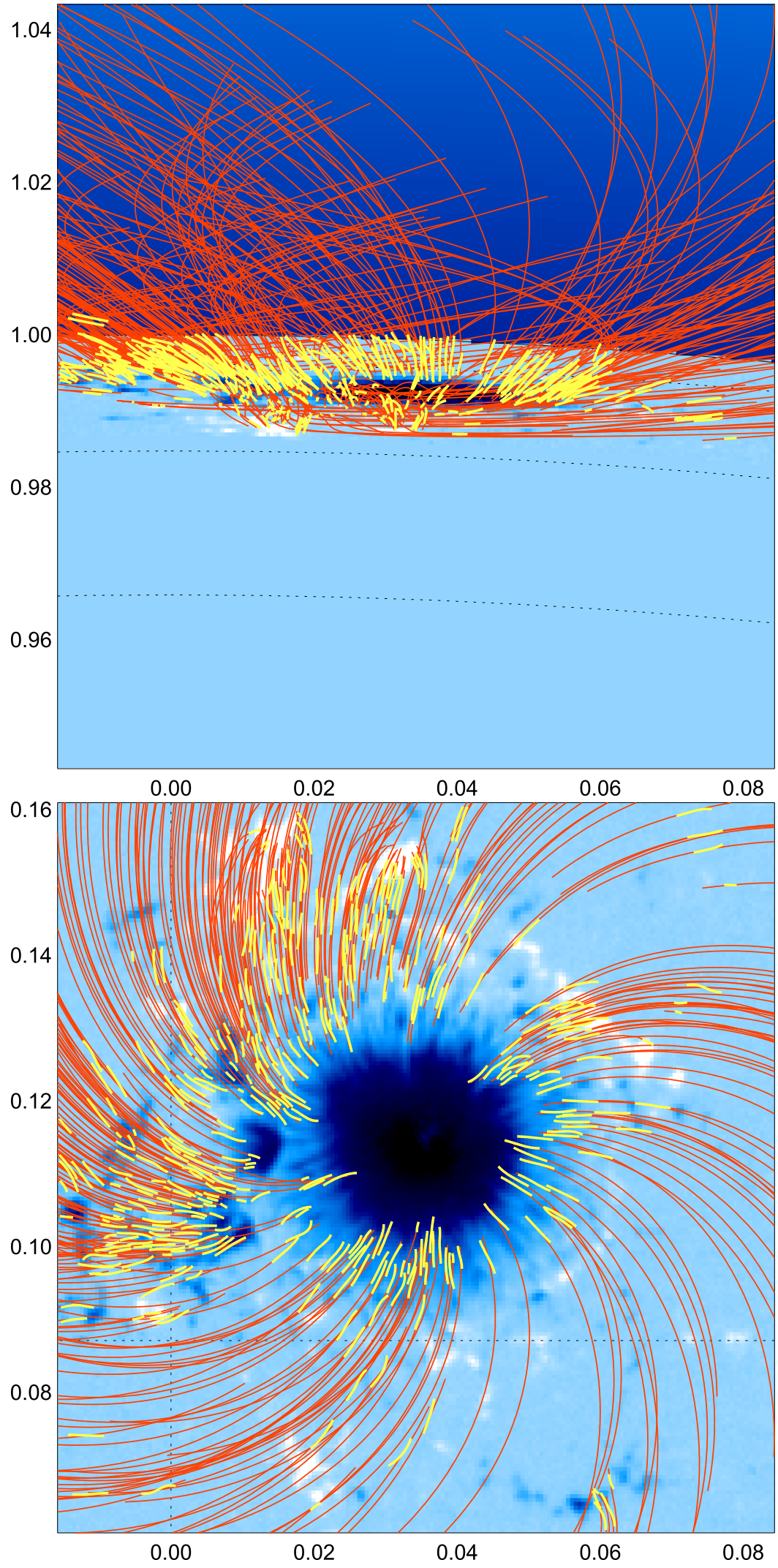

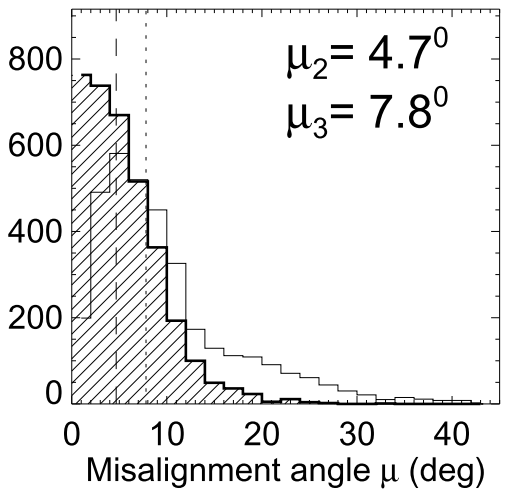

INPUT:

Instrument $\quad=|B| S$

NOAA $=11092$, N12W02

Wavelengths $=8542 \mathrm{~A}$

fov $\quad=0.100$

amis $\quad=20.0$

nsm1 $=1$

nmag_p, $\mathrm{np}=30,30$

nstruc $\quad=1000$

nitmin, nitmax $=40,100$

prox_min $=10.0$

Imin,rmin $=5,8$

qthresh1,2 $=0.00,0.00$

OUTPUT:

$[x 1, x 2] \quad=-0.0159,0.0841$

[y1,y2] $=0.0608,0.1608$

dpix HMI $=0.0005,0.51$ arcsec

dpix IBIS $=0.0001,0.10$ arcsec

nloop,ndet $=386 / 703$

nloop $/$ ndet $=0.549$

misalign $\quad=4.7,7.8 \mathrm{deg}$

div-free $\quad=1.2 \mathrm{e}-02$

force-free $\quad=1.9 \mathrm{e}-02$

weight curr $=9.9 \mathrm{e}-01$

qe_rebin $=0.985$

qe_model $=1.014$

qiso_corr $=2.467$

E_P $\quad=581.3 \times 10^{30} \mathrm{erg}$

$E_{\text {free }} \quad=92.5 \times 10^{30} \mathrm{erg}$

E_NP/E_P $=1.159$

Iterations $=53$

$\mathrm{CPU}=339.4 \mathrm{~s}$

Figure 7. Automated feature tracking is applied to the chromospheric IBIS image (2010 August 03, 15:23 UT) in the wavelength of Ca II $8542 \AA$ wavelength, otherwise similar representation as in Figure 5. 


\section{3_152300, time step=1, IBIS H-alpha 6563 A}
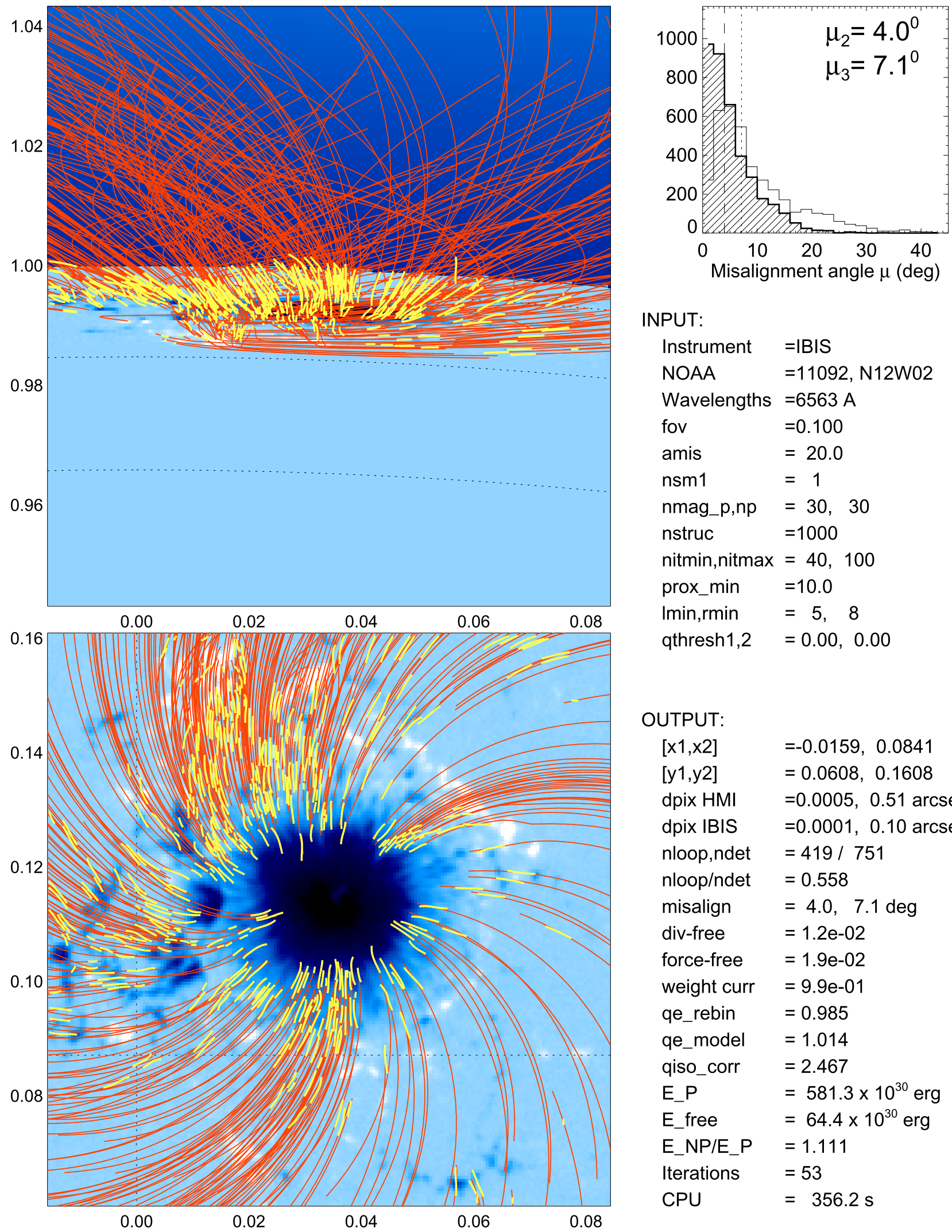

INPUT:

$\begin{array}{ll}\text { Instrument } & =I B I S \\ \text { NOAA } & =11092, \text { N12W02 } \\ \text { Wavelengths } & =6563 \mathrm{~A} \\ \text { fov } & =0.100 \\ \text { amis } & =20.0 \\ \text { nsm1 } & =1 \\ \text { nmag_p,np } & =30,30 \\ \text { nstruc } & =1000 \\ \text { nitmin,nitmax } & =40,100 \\ \text { prox_min } & =10.0 \\ \text { Imin,rmin } & =5, \quad 8 \\ \text { qthresh1,2 } & =0.00,0.00\end{array}$

OUTPUT:

$[\mathrm{x} 1, \mathrm{x} 2] \quad=-0.0159,0.0841$

$[\mathrm{y} 1, \mathrm{y} 2] \quad=0.0608,0.1608$

dpix HMI $=0.0005,0.51$ arcsec

dpix IBIS $=0.0001,0.10$ arcsec

nloop,ndet $=419 / 751$

nloop/ndet $=0.558$

misalign $\quad=4.0,7.1 \mathrm{deg}$

div-free $\quad=1.2 \mathrm{e}-02$

force-free $\quad=1.9 \mathrm{e}-02$

weight curr $=9.9 \mathrm{e}-01$

qe_rebin $\quad=0.985$

qe_model $=1.014$

qiso_corr $=2.467$

E_P $\quad=581.3 \times 10^{30} \mathrm{erg}$

$E_{\text {free }} \quad=64.4 \times 10^{30} \mathrm{erg}$

E_NP/E_P $=1.111$

Iterations $\quad=53$

$\mathrm{CPU} \quad=356.2 \mathrm{~s}$

Figure 8. Automated feature tracking is applied to the chromospheric IBIS image (2010 August 03, 15:23 UT) in the wavelength of H $\alpha 563 \AA$ wavelength, otherwise similar representation as in Figure 5. 
Table 3

Data Analysis Results of the Number of Detected (Field-aligned) Loops $n_{\text {det }}$, Fitted Loops $n_{\text {loop }}$, the 2D and 3D Misalignment Angles $\mu_{2}$ and $\mu_{3}$, the Potential Energy $E_{\mathrm{P}}$, and the Ratio of the Free Energy $q_{\text {free }}=E_{\mathrm{NP}} / E_{\mathrm{P}}-1$

\begin{tabular}{|c|c|c|c|c|c|c|c|}
\hline $\begin{array}{l}\text { Observation } \\
\text { date }\end{array}$ & Instrument & $\begin{array}{c}\text { Detected } \\
\text { loops } \\
n_{\mathrm{det}} \\
\end{array}$ & $\begin{array}{c}\text { Fitted } \\
\text { loops } \\
n_{\text {loop }} \\
\end{array}$ & $\begin{array}{c}\text { Misalignment } \\
\text { angle 2D } \\
\mu_{2}(\mathrm{deg})\end{array}$ & $\begin{array}{c}\text { Misalignment } \\
\text { angle 3D } \\
\mu_{3}(\mathrm{deg})\end{array}$ & $\begin{array}{c}\text { Potential } \\
\text { energy } \\
E_{\mathrm{P}}\left(10^{30} \mathrm{erg}\right)\end{array}$ & $\begin{array}{c}\text { Free energy } \\
\text { ratio } \\
q_{\text {free }} \\
\end{array}$ \\
\hline 2010 Aug 03 & AIA $171+$ & $222 \pm 76$ & $167 \pm 36$ & $5^{\circ} 0 \pm 3.2$ & $7^{\circ} .8 \pm 0.5$ & 571 & $0.03 \pm 0.01$ \\
\hline 2010 Aug 03 & IBIS 8542 & $656 \pm 121$ & $338 \pm 62$ & $4^{\circ} .0 \pm 0.6$ & $7.1 \pm 0.7$ & 571 & $0.13 \pm 0.04$ \\
\hline 2010 Aug 03 & IBIS 6563 & $712 \pm 114$ & $421 \pm 75$ & $4^{\circ} .0 \pm 0.4$ & $7.2 \pm 0.8$ & 571 & $0.11 \pm 0.01$ \\
\hline 2014 Aug 24 & AIA 304 & $45 \pm 21$ & $17 \pm 6$ & $7.4 \pm 2.2$ & $7.5 \pm 1.5$ & 551 & $0.11 \pm 0.05$ \\
\hline 2014 Aug 24 & ROSA 6563 & $654 \pm 98$ & $232 \pm 75$ & $6.5 \pm 1: 3$ & $8.5 \pm 1.8$ & 551 & $0.26 \pm 0.01$ \\
\hline 2014 Aug 30 & AIA $171+$ & $190 \pm 87$ & $83 \pm 34$ & $5.4 \pm 1.2$ & $10^{\circ} .9 \pm 2.4$ & 559 & $0.10 \pm 0.05$ \\
\hline 2014 Aug 30 & AIA 304 & $43 \pm 22$ & $16 \pm 7$ & $7^{\circ} .0 \pm 1.0$ & $10^{\circ} .7 \pm 2 . .4$ & 559 & $0.10 \pm 0.09$ \\
\hline
\end{tabular}

Note. The means and error bars are averaged from fitting 5 different variations of the control parameters.

and $\mathrm{H}$-alpha $6563 \AA$ data yield a significantly larger value of $q_{\text {free }}=13 \% \pm 4 \%$, and $q_{\text {free }}=11 \% \pm 1 \%$ (Table 3), respectively. It is remarkable that the free energies measured with Ca II $8542 \AA$ and $\mathrm{H} \alpha 6563 \AA$ agree within $20 \%$, which indicates that the traced structures are nearly identical, radiate at a similar temperature, and originate in similar chromospheric heights. For a direct comparison of field-aligned structures see Figure 9. The traced structures appear to be preferentially rooted in the penumbra, are absent in the umbra, and are sparse outside the penumbra. We performed also measurements with AIA $304 \AA$ data and obtained a value of $q_{\text {free }}=6 \% \pm 3 \%$ that is intermediate to the relatively higher free energy in the chromosphere and the relatively lower free energy in the corona. Since the coronal data lead to a significantly lower amount of free energy than the chromospheric data, we conclude that the two datasets are susceptible to different subsets of magnetic field lines, which are governed by different degrees of non-potentiality. Chromospheric fibrils and footpoints of loops apparently reveal a higher degree of helical twist than coronal loops.

The second case (NOAA 12146 observed on 2014 August 24) exhibits a free energy ratio of $q_{\text {free }}=26 \% \pm 1 \%$ (Table 3) from the chromospheric ROSA H-alpha image, while the values from the coronal AIA data are lower also, but somewhat less reliable, given the relatively large misalignment angle of $\mu_{2} \gtrsim 6^{\circ}$ that indicates insufficient convergence, either due to a lack of suitable loops, due to the complexity of nested loops, or due to confusing projection effects at a heliographic longitude of W25.

The third case (NOAA 12149 observed on 2014 August 30) shows a free energy ratio of of $q_{\text {free }}=26 \% \pm 1 \%$ from the chromospheric IRIS $2796 \AA$ data, and of $q_{\text {free }}=17 \% \pm 4 \%$ from the chromospheric ROSA H $\alpha$ data, while the coronal AIA data show again a lower value of $q_{\text {free }}=10 \% \pm 5 \%$ (Table 3 ).

In summary, we find that the coronal data tend to underestimate the total free energy of an active region in all three cases, by a typical factor of $\approx 2-4$. The highest accuracy or smallest misalignment angle is achieved for heliographic positions near disk center $\left(\mu_{2} \approx 4^{\circ}\right)$, while heliographic positions all the way to a distance of $0.7 R_{\odot}$ become gradually less accurate $\left(\mu_{2} \approx 7^{\circ}\right)$. This may indicate that the 3D geometry appears to be more nested and confused near the limb, imposing a bigger challenge for accurate 3D reconstruction. In addition, the contrast of the structures in the H-alpha images are higher in the IBIS images than in the ROSA images due to the narrower passband of IBIS. Nevertheless, since we achieve a similar accuracy for the best-fit median misalignment angle $\left(\mu_{2}, \mu_{3}\right)$ for both chromospheric structures and coronal loops we can conclude that chromospheric fibrils (or footpoints of coronal loop structures) are generally field-aligned, within an accuracy of $\mu_{2} \approx 4^{\circ}-7^{\circ}$.

\subsection{Altitudes of Chromospheric Tracers}

Our VCA-NLFFF magnetic field model is a space-filling 3D model that fulfills the divergence-freeness and force-freeness, but the forward-fitting is an application to 2D data, namely the projected 2D coordinates $[x(s), y(s)]$ of the automatically traced loops. In principle one could forward-fit also to 3D data, if stereoscopic observations are available (e.g., see comparison of 2D versus 3D forward-fitting in Aschwanden 2013c). Since the best-fit solution of our VCA-NLFFF code provides the LOS coordinate $z$ for every loop tracing position $(x, y)$, we can directly determine the altitude range $h=r-1=$ $\sqrt{x^{2}+y^{2}+z^{2}}-1$ for each traced loop segment. This allows us to measure an altitude distribution of all traced loop segments in a given wavelength range, which corresponds to the contribution function (as a function of height) in each wavelength. An approximate range of the altitude distribution of coronal and chromospheric tracers can already be seen in the side views shown in Figures 5-8. Coronal loop tracings cover most of the altitude range of $h \lesssim 0.05 R_{\odot}=35,000 \mathrm{~km}$ (Figures 5 and 6 top panels), while chromospheric tracers extend only over a chromospheric height range of $h \lesssim 0.005 R_{\odot}=3500 \mathrm{~km}$ (Figures 7 and 8, top panels), although we used an identical computation box with a height range of $h_{\max }=0.05 R_{\odot}=35,000 \mathrm{~km}$ in fitting each data set. 

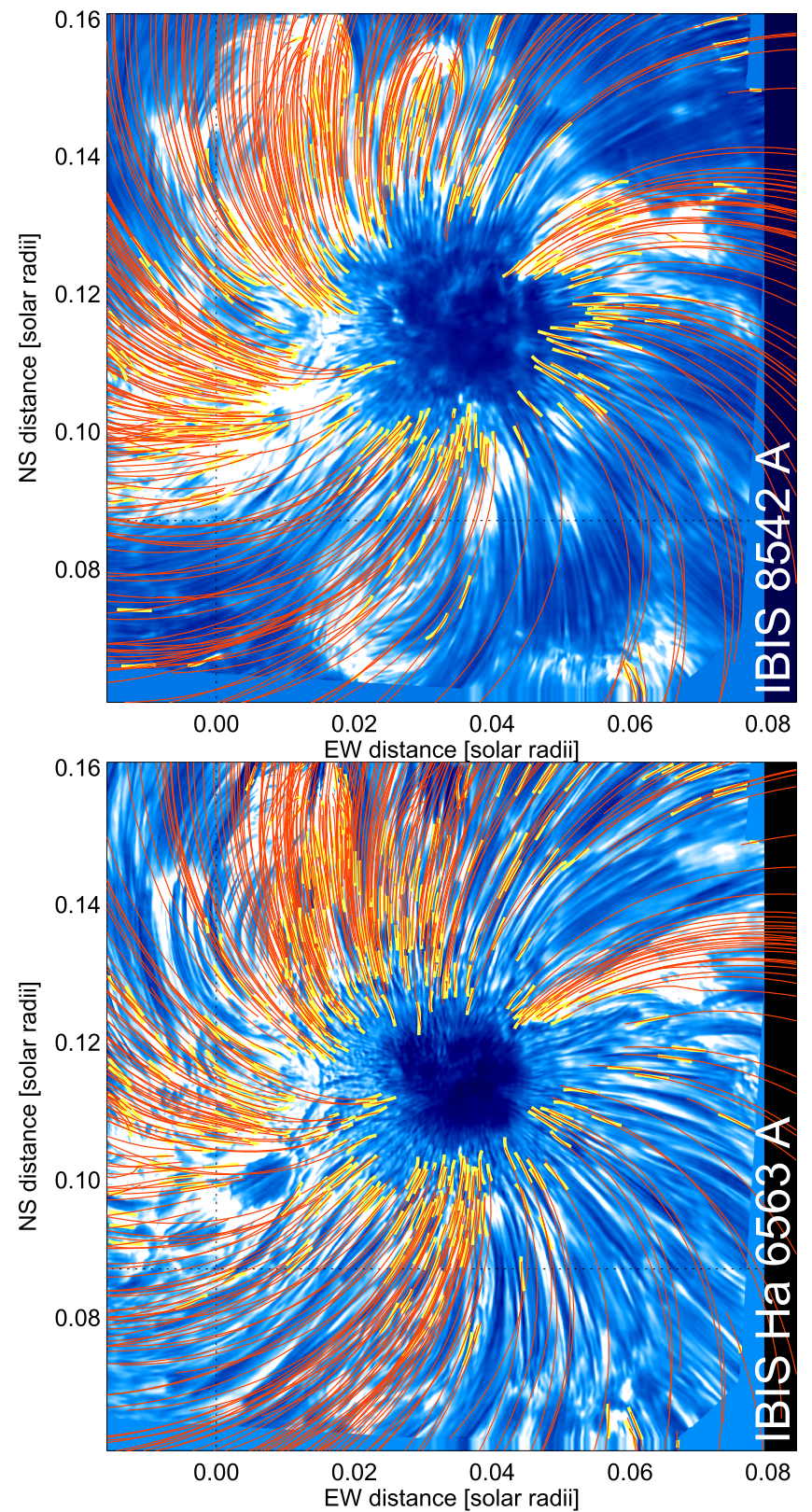

Figure 9. A subset of automatically traced loop segments (yellow curves) and best-fit magnetic field lines (red curves) are shown, overlaid on the IBIS $8542 \AA$ image (top frame) and $\mathrm{H} \alpha 6563 \AA$ image (bottom frame) in which the automated tracing was performed.

We plot histograms of the altitudes $h$ of all automatically traced loop segments (or chromospheric features) for each instrument or wavelength separately, where each segment with a length of $\Delta s=0.002 R_{\odot}$ is counted as an individual element, which is shown in Figure 10. Interestingly, both the hot coronal structures detected with AIA mostly in 171 and $193 \AA$, as well as the cooler structures detected with AIA at $304 \AA$ are found to have coronal altitudes. It appears that the He II $304 \AA$ line traces a lot of cool plasma in coronal structures, either from static cool loops, or from cooling loops that produce "coronal rain," or cool plasma of quiescent prominence and filaments, rather than cool plasma in chromospheric heights. On the other side, the IBIS $8542 \AA$, the ROSA H $\alpha 6563 \AA$, and the IRIS Mg II $h / k$

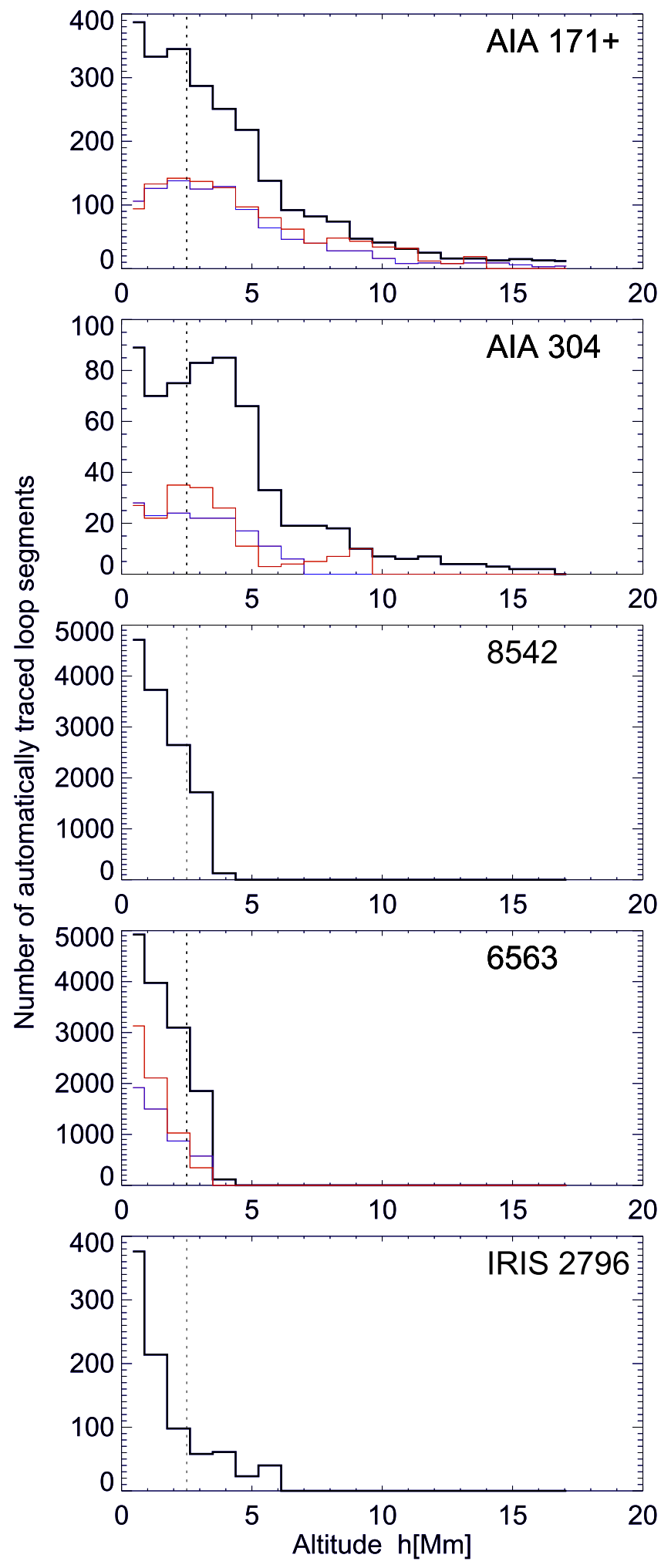

Figure 10. Distribution function of altitudes $h$ of automatically traced curvilinear elements according to the 3D modeling of the forward-fitting VCANLFFF code. Most of the features seen with IBIS, ROSA, and IRIS originate in the chromosphere (marked at a nominal height of $2500 \mathrm{~km}$ with a dotted line) and transition region, while most of the structures seen with AIA belong to the corona, including the emission at $304 \AA$. The distributions are measured in 3 different active regions (Table 2): for NOAA 11092 on 2010 August 3 (black), for NOAA 12146 on 2014 August 24 (blue), and for NOAA 12149 on 2014 August 30 (red). 
line detect cool plasma only in the chromosphere or lower transition region with an upper limit of $h \lesssim 4000 \mathrm{~km}$. It appears that our VCA-NLFFF magnetic modeling method is equally suited to measure altitudes of chromospheric and coronal features as stereoscopic and tomographic methods (e.g., see review of Aschwanden 2011).

\subsection{Plasma- $\beta$ Parameter of Chromospheric Tracers}

Our VCA-NLFFF model provides the magnetic field strength $B$ in each automatically traced curvi-linear feature, be it a coronal loop or a chromospheric fibril. Assuming pressure balance in the chromosphere, we can then use a hydrostatic chromospheric density $n_{e}(h)$ and temperature model $T_{e}(h)$ in order to determine the plasma- $\beta$ parameter as a function of height $h$,

$$
\beta(h)=\frac{p_{\mathrm{th}}(h)}{p_{\mathrm{mag}}(h)}=5.2 \times 10^{-15} \zeta n(h) T_{e}(h) B(h)^{-2},
$$

where $\zeta=1$ is the ionization fraction. The gas pressure is estimated from $p_{\mathrm{th}}(h)=(3 / 2) n(h) k_{B} T_{e}(h)$, with the particle density $n=n_{e}+1.1\left(n_{\mathrm{H} i}+n_{\mathrm{H} i i}\right)$, composed of electrons, neutral and ionized hydrogen, where the factor 1.1 accounts for all other elements. For a coronal density of $n_{e} \approx 10^{9} \mathrm{~cm}^{-3}$ and a coronal temperature of $T_{e} \approx 10^{6} \mathrm{~K}$ we thus expect for magnetic field strength in the range of $B \approx 100-1000 \mathrm{G}$ a fairly low value of $\beta \approx 10^{-3}-10^{-5}$, which explains the perfect plasma confinement in the corona, that holds for most of the upper chromosphere also (Gary 2001).

Using the VAL-C model (Vernazza et al. 1981; Fontenla et al. 1990, 1993) for the chromosphere in a range of $h \approx 0-2000 \mathrm{~km}$ and the coronal canopy model of Gabriel (1976) in the height range of $h=2000-100,000 \mathrm{~km}$, we calculate the plasma- $\beta$ parameter $\beta(h)$ as a function of the height $h$, based on our magnetic field solutions $B(h)$ for each automatically traced loop or chromospheric feature. We show the results in Figure 11 and see that the magnetic field strength in all traced features varies in the range of $B \approx 100-1000 \mathrm{G}$ (Figure 11, left panels). Of course, there are areas with lower field strengths outside of the analyzed sunspots and active regions, but it appears that all (automatically) traced loop structures and fibrils are anchored in strong-field regions, while we find virtually no loop rooted in areas with $B \lesssim 50 \mathrm{G}$, a finding that is also consistent with the $\beta=1$ contour outside the penumbral region in another case (Jess et al. 2013, Figure 1 therein). This result in itself may have important consequences for coronal heating models. Using then these field strengths $B$ (h) and combining with the temperature $T_{e}(h)$ and density models $n_{e}(h)$ we obtain then with Equation (1) the variation of the plasma- $\beta$ parameter with height, which is shown in the Figure 11 (right-hand panels). Apparently the values of the plasma- $\beta$ parameter are below unity for all traced loop structures, spreading over a height range of $h \approx 100-20,000 \mathrm{~km}$. This applies not only to coronal loops, but also to all chromospheric structures (fibrils) observed here with IBIS, ROSA, and IRIS. The observed preference for low plasma- $\beta$ values implies locations inside magnetic field concentrations, which does not exclude higher plasma- $\beta$ values outside the observed structures. Our observational finding is also consistent with the theoretical work of Leenaarts et al. (2012; Figure 14 therein), which finds the formation of the $\mathrm{H} \alpha$ line in the chromosphere in regions with $\beta<0.1$.

\section{DISCUSSION}

\subsection{Chromosphere Suitability for Magnetic Modeling}

The major motivation for this study is the question whether chromospheric data are useful for magnetic field modeling. From previous modeling we know that coronal data that show the geometry of loops are highly useful for NLFFF modeling. DeRosa et al. (2015) conclude in their latest NLFFF modeling comparison: "We continue to recommend verifying agreement between the modeled field lines and corresponding coronal loop images before any NLFFF model is used in a scientific setting." On the other side we are painfully aware of the limitations of photospheric data, regarding the actual nonforcefreeness that violates the forcefree assumption used in NLFFF extrapolations from the photospheric boundary (DeRosa et al. 2009). So, what about the chromosphere, which is situated in the interface between the photosphere and the corona? In particular to cicrumvent the forcefreeness dilemma, the question was posed by Wiegelmann: "Can we improve the preprocessing of photospheric magnetograms by the inclusion of chromospheric observations?" (Wiegelmann et al. 2008). This idea was tested with a model image of $\mathrm{H} \alpha$ fibrils and was found to improve the NLFFF solutions in a model chromosphere (Metcalf et al. 2008; Wiegelmann et al. 2008).

Since the solar atmosphere was found to be force-free above $h \approx 400 \mathrm{~km}$ in active regions (Metcalf et al. 1995), the forcefreeness is not violated in the chromosphere. Since the altitudes of chromospheric structures observed with IBIS, IRIS, and ROSA is consistent with a height range of $h \approx 400-4000 \mathrm{~km}$ as measured with our VCA-NLFFF code (Figure 11), and moreover the plasma- $\beta$ parameter was found to be less than unity in the entire height range of $h \approx 100-35,000 \mathrm{~km}$, chromospheric features should be field-aligned as well as not violate the force-freeness condition, a finding that is consistent with another sunspot study (Jess et al. 2013; Figure 1 therein). Another confirmation that chromospheric features are fieldaligned is corroborated with our finding that the automatically traced chromospheric features (fibrils and footpoints of loops) match the best-fit VCA-NLFFF solutions as close as coronal data do, for instance the 2D misalignment for chromospheric data is about $\mu_{2} \gtrsim 4^{\circ}$ (Table 3 ). This match is even more accurate than the field-alignment of super-penumbral fibrils measured with IBIS $8542 \AA$ determined with a Hanle and Zeeman modeling code, which was found to be $\mu_{2} \approx 10^{\circ}$ (Schad et al. 2013), or with SPINOR/CRISP observations, which show a median misalignment of $\mu \approx 20^{\circ}$ (de la Cruz Rodriguez \& Socas-Navarro 2011; their Table 2). However, since their quoted errors are of similar magnitude as the misalignment angles themselves, their results may well be consistent with the notion that fibrils are field-aligned.

Thus, at a first glance, we can answer the question of the suitability of chromospheric data for magnetic modeling clearly in an affirmative way, based on their force-free nature, their low plasma- $\beta$ parameter, and the small misalignment angle range found with the best-fit VCA-NLFFF solutions. However, we have to add a caveat that our measurements mostly apply to strong-field regions while the appearance of field-aligned fibrils may be more chaotic and less force-free in weak-field regions such as in the Quiet Sun, rendering chromospheric data possibly less useful for magnetic modeling there (Wiegelmann et al. 2015). 

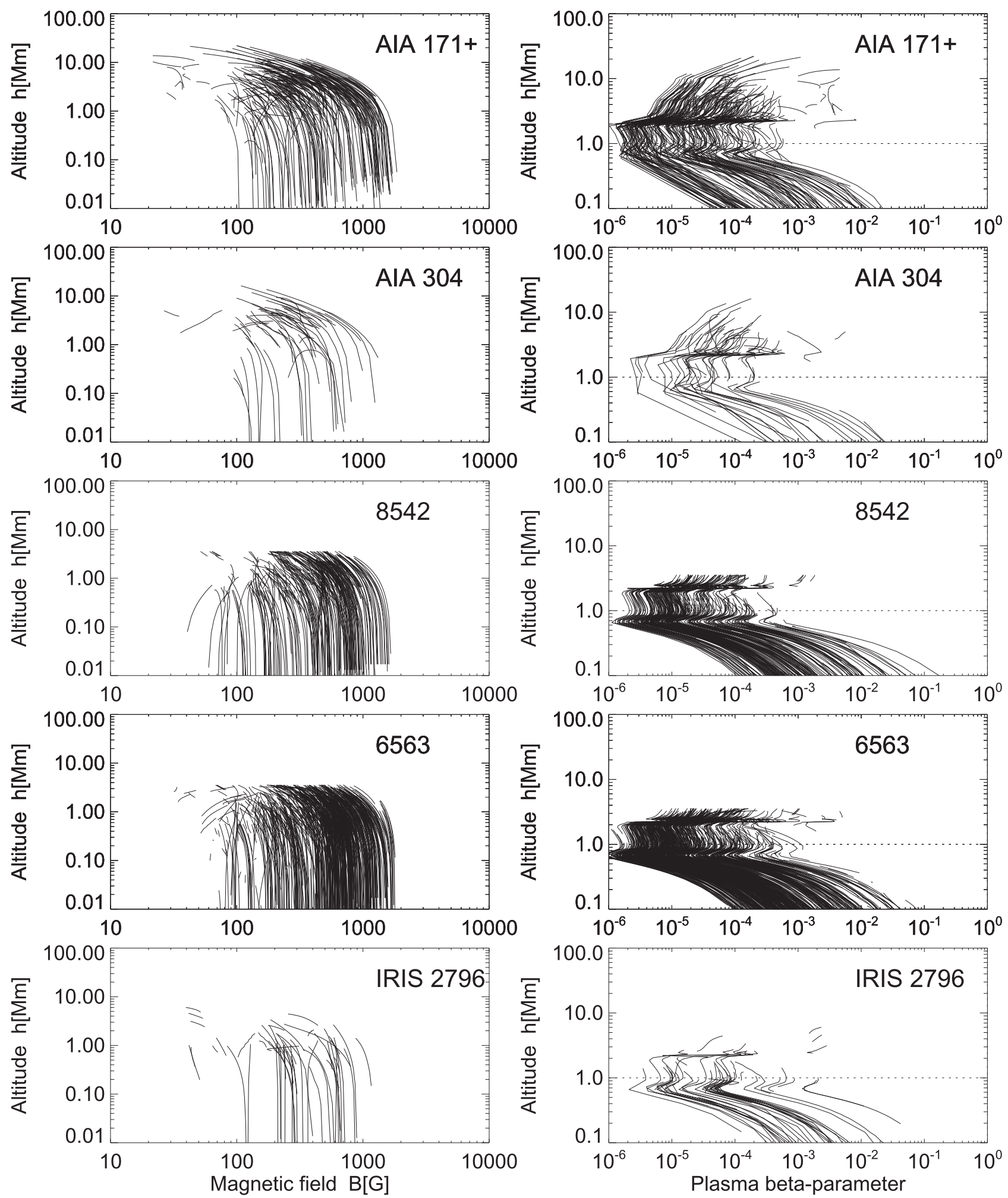

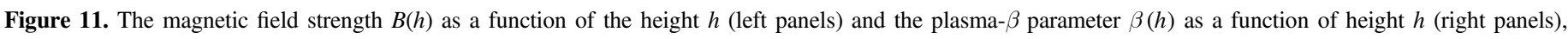

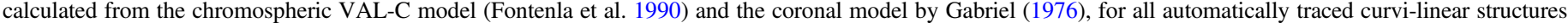
observed with each instrument (AIA, IRIS, IBIS, ROSA).

\subsection{Chromospheric versus Coronal Free Energy}

Ideally, the volume-integrated free energy in an active region should match for chromospheric and coronal data, if a perfect coronal magnetic field model is available. A good correspondence of the free energy between chromospheric data (IRIS,
AIA 304, $1600 \AA$ ) and coronal data (AIA 94, 131, 171, 193, $211,335 \AA$ ) was indeed found during a solar flare, where the time evolution of the free energy agreed in magnitude as well as exhibited a synchronized increase and decrease in coronal and chromospheric data (Aschwanden 2015), based on an earlier version of the VCA-NLFFF code used here. 
In the present study we notice a substantial disagreement between the free energy of an active region computed from chromospheric versus coronal tracers, where the coronal data reveal a trend to underestimate the free energy. Obviously, the two data sets with automatically traced structures do not constitute representative samples, but rather may contain mutually exclusive fractions, one being sampled in the chromosphere at altitudes of $\lesssim 4000 \mathrm{~km}$, while the other is sampled in the corona up to $h \lesssim 35,000 \mathrm{~km}$. The two samples appear to have a different degree of magnetic twist, and therefore yield a different amount of free energy. The chromosphere seems to contain stronger twisted loops than the corona, which results into a higher free energy. In future applications we might combine the chromospheric and coronal data in a single forward-fitting procedure, so that a more complete and more representative sample of field-aligned structures is available. For the time being we have to conclude that the coronal data can underestimate the free energy (as determined with the VCA-NLFFF code), while the chromospheric data appear to sample the magnetic field more comprehensively.

\subsection{Chromospheric Height Contribution Function}

Understanding the 3D geometry of chromospheric structures (fibrils, loops, moss (Berger et al. 1999, etc.)) in the context of 3D magnetic field modeling requires the height contribution function of each observed wavelength. The atmospheric height where the contribution function to the intensity has a maximum strongly varies from the line core to the line wings. Measurements of the chromospheric magnetic field at several wavelengths within the $\mathrm{Na}$ I line, by computing the net Lorentz force as a function of wavelength (using the equations of Molodenskii 1969) demonstrated the height dependence of the force, since the wings of the $\mathrm{Na}$ I line are formed deeper in the atmosphere than the core (Metcalf et al. 1995). Combining this magnetic height dependence $B(h)$ with an atmospheric model of the density and temperature (VAL-F model), the contribution function of the $\mathrm{Na}$ I line was found to extend over a height range of $h \approx 100-1000 \mathrm{~km}$ (Figure 6 of Metcalf et al. 1995). For the $\mathrm{Ca}$ II $8542 \AA$ line, the best correlation between a chromospheric magnetogram and a magnetic potential field model was found at $800 \mathrm{~km}$, which represents the peak of the line formation (Choudhary et al. 2001; Leenaarts et al. 2009).

The Ca II $8542 \AA$ contribution function was also calculated with the FAL-C model (Fontenla et al. 1993) and with hydrodynamic simulations by Carlsson \& Stein (1997), yielding a range of $h \approx 300-1000 \mathrm{~km}$ for the bulk of the Ca II emission (Figure 1 in Pietarila et al. 2009), and $h \lesssim 1300 \mathrm{~km}$ (Figure 5 in Cauzzi et al. 2008). Comparing these height ranges with our 3D magnetic field reconstruction (with VCA-NLFFF), we find a somewhat larger height range of $h \lesssim 4000 \mathrm{~km}$ for the total of all automatically traced curvi-linear features in IBIS, ROSA, and IRIS data, which may be explained by dynamic phenomena that populate the upper chromosphere of $h \approx 2000-4000 \mathrm{~km}$, in excess of the static chromospheric models that span over a lower height of the chromosphere ( $h \lesssim 2000 \mathrm{~km}$ for VAL and FAL models). However, the extended chromosphere is filled with ubiquitous spicules and fibrils up to $h \lesssim 5000 \mathrm{~km}$, according to radio, hard X-ray, and and UV observations (Ewell et al. 1993; Aschwanden et al. 2002). H $\alpha$ filtergrams show fibrils up to heights of $h \approx 3000-4000 \mathrm{~km}$ (Harvey et al. 1999; Choudhary et al. 2001). In any case, 3D magnetic field modeling provides height information that is complementary to the contribution functions determined with hydrodynamic models. On the other side, (M)HD models and (N)LTE radiative transfer calculations can provide information on the contribution functions in a statistical manner.

\section{CONCLUSIONS}

We explored the suitability of chromospheric images for magnetic modeling, using high-resolution images from IBIS and ROSA in the Ca II line at $8542 \AA$, and in the $\mathrm{H} \alpha 6563 \AA$ line, and from the IRIS $\mathrm{Mg}$ II line, and compared the results with coronal images taken with AIA/SDO. Our investigation made use of a novel magnetic field calculation method by fitting field lines that are parameterized by an approximative solution of the NLFFF based on a vertical-current approximation (the so-called VCA-NLFFF code). The field lines of the theoretical model are forward-fitted to curvi-linear (looplike) structures that are automatically detected with the OCCULT-2 code in chromospheric or coronal images. We applied this VCA-NLFFF code to observations of active regions observed on three days (2010 August 3; 2014 August 24 and 30) during IBIS and ROSA campaigns. Since the previous application of the VCA-NLFFF code to coronal images from TRACE and AIA has proven the suitability of coronal images for magnetic field reconstruction methods, we aim to test here the same application to chromospheric images. Our conclusions from this first exploration of chromospheric data in this context are as follows:

1. The suitability of chromospheric images for magnetic modeling: Chromospheric images with high spatial resolution (0." $2-0$ !' 3$)$, especially from the core of the Ca II line (8542 $\AA$ ) and from the core of the $\mathrm{H} \alpha$ line (6563 $\AA$ ), reveal a wealth of crisp curvi-linear structures (fibrils and loop segments) and thus are well-suited to constrain magnetic field solutions. The suitability has been measured in terms of the $2 \mathrm{D}$ and $3 \mathrm{D}$ misalignment angle between the magnetic field model and the observed field directions, as obtained from automated detection of curvi-linear structures. For the best case of a sunspot region near the center of the solar disk, a misalignment angle of $\mu_{2} \approx 4^{\circ}$ was achieved for a chromospheric image (from IBIS), while AIA data yield a similar value $\left(\mu_{2} \approx 5^{\circ}\right)$. Other cases at a larger distance to disk center (at W25 and W44) yielded less accurate values $\left(\mu_{2} \approx 5^{\circ}-7^{\circ}\right)$. This work seems to indicate that the orientation of the fibrils seen in the chromospheric images seem to be consistent with a realistic model of the magnetic field in the chromosphere, which is in contrast to previous work that showed occasional misalignments between fibrils and magnetic field models (de la Cruz Rodriguez \& Socas-Navarro 2011; Schad et al. 2013).

2. The free(magnetic) energy in an active region, i.e., the difference between the non-potential and potential magnetic energy, is an important upper limit for the global energy that can be dissipated in a flare or CME. For the most accurate of our three cases we found a chromospheric free energy ratio of $q_{\text {free }}=13 \% \pm 4 \%$ and $q_{\text {free }}=11 \% \pm 1 \%$. These values correspond to the magnetic energy integrated over a computation box with a $\mathrm{FOV}$ of $0.1 R_{\odot}$, where the total potential energy is 
$E_{\mathrm{P}}=5.71 \times 10^{32} \mathrm{erg}$. These values are significantly higher than what we find from coronal tracers in the same region, i.e., $q_{\text {free }}=3 \% \pm 1 \%$, using combined AIA images at the wavelengths of $94-335 \AA$. Thus, the chromospheric data probe a higher degree of nonpotential, helically twisted structures (fibrils and loop footpoint segments) at chromospheric temperatures, while the loops imaged in coronal temperatures appear to be less twisted and underestimate the total free energy of the active region by a factor of $\approx 4$ in this case. In the other two cases we analyzed, which are further away from the solar disk center and provide less accurate measurements of magnetic energies, we find that AIA underestimates the free energy derived from ROSA by factors of 1.5-2.5, and thus reveal the same trend.

3. Contribution function of altitude: The 3D magnetic field model of the VCA-NLFFF code yields also a fit of the LOS coordinate $z(s)$ to each curvi-linear loop segment that is traced in the $[x, y]$ plane with the OCCULT-2 code. From the so obtained 3D coordinates $[x, y, z]$ we can immediately derive the altitude $h$ of each traced loop segment. Plotting histograms of these altitudes we find that the IBIS, ROSA, and IRIS data all reveal chromospheric and transition region structures in a height range of $h \lesssim 4000 \mathrm{~km}$, which is consistent with the height range of fibrils, filaments, mottles, and spicules in the dynamic part of the chromosphere, which extend beyond the hydrostatic height range of $h \lesssim 2000 \mathrm{~km}$. In contrast, the AIA 171, 193, $211 \AA$, as well as the AIA $304 \AA$ wavelength in the cooler $\mathrm{He}$ II line reveal cool plasma structures in a height range of $h \lesssim 35,000 \mathrm{~km}$. Apparently, the features seen in $\mathrm{He}$ II include mostly cool plasma at coronal heights, including "coronal rain," filaments, and prominences, rather than structures in the chromosphere.

4. Plasma $\beta$-parameter: From our 3D magnetic field model obtained with VCA-NLFFF we can also directly calculate the magnetic field $B(h)$ as a function of height for each traced structure, as well as the plasma- $\beta$ parameter $\beta(h)$ as a function of height. Interestingly we find that all traced structures have magnetic fields in the range of $B(h) \approx 100-1000 \mathrm{G}$, and a plasma- $\beta$ parameter in the range of $\beta(h) \approx 10^{-5}-10^{-1}$, over the entire mapped height range of $h \approx 100-35,000 \mathrm{~km}$. Thus all traced structures are magnetically confined, which can be explained by their proximity to the dominant sunspot. However, it is surprising that our automated feature detection code did not pick up any structure (out of the $\approx 1500$ field-aligned structures found in all fits) in regions with a low magnetic field, and possibly with a plasma- $\beta$ parameter in excess of unity. This finding has perhaps the important consequence that coronal heating occurs mostly in strong-field regions with $B \gtrsim 100 \mathrm{G}$, rather than in weak-field regions.

In summary, our study has shown that high-resolution chromospheric images are extremely useful for magnetic modeling, equally important as (high-resolution) coronal images. Obviously, the optimum wavelengths are in the core of UV and $\mathrm{H}-\alpha$ line profiles, which have a peak of the height contribution function in the upper chromosphere. The features that constrain magnetic field models best are crisp curvi-linear structures, such as loops, fibrils, filaments, spicules, and threads of prominences. We learned that chromospheric features may even yield more comprehensive estimates of the free magnetic energy than coronal loops, and thus both data sets should be combined for magnetic modeling in future efforts. The features seen in chromospheric images complement those seen in coronal images, which probe two different but complementary height ranges. The reliability of any (non-potential) magnetic field solution obtained with either chromospheric or coronal images depends strongly on the selection of traced features, which should ideally comprise a representative subset of all magnetic field structures in a given computation box of an active region, sampled in both the chromospheric and coronal height range. All these conclusions strongly suggest that chromospheric and coronal data need to be quantitatively included in magnetic field models of solar flares and active regions, a requirement that is incorporated in the present VCANLFFF method, or in the quasi-Grad-Rubin method of Malanushenko et al. (2014), while there is no provision for such a capability in traditional NLFFF codes.

The authors are indebted to the constructive comments of an anonymous referee, and to helpful discussions with Bart DePontieu, Mark DeRosa, Anna Malanushenko, Carolus Schrijver, Alberto Sainz-Dalda, Ada Ortiz, and Jorrit Leenaarts. Part of the work was supported by the NASA contracts NNG04EA00C of the SDO/AIA instrument and NNG09FA40C of the IRIS mission. D.B.J. thanks the UK's Science and Technology Facilities Council (STFC) for an Ernest Rutherford Fellowship, in addition to a dedicated standard grant, which allowed this project to be undertaken.

\section{REFERENCES}

Amari, T., Boulmezaoud, T. Z., \& Aly, J. J. 2006, A\&A, 446, 691

Aschwanden, M. J. 2010, SoPh, 262, 399

Aschwanden, M. J. 2011, LRSP, 8, 5

Aschwanden, M. J. 2013a, SoPh, 287, 323

Aschwanden, M. J. 2013b, SoPh, 287, 369

Aschwanden, M. J. 2013c, ApJ, 763, 115

Aschwanden, M. J. 2015, ApJL, 804, L20

Aschwanden, M. J. 2016, ApJS, 224, 25

Aschwanden, M. J., Brown, J. C., \& Kontar, E. P. 2002, SoPh, 210, 383

Aschwanden, M. J., De Pontieu, B., \& Katrukha, E. A. 2013, Entrp, 15, 3007

Aschwanden, M. J., Lee, J. K., Gary, G. A., Smith, M., \& Inhester, B. 2008 , SP, 248, 359

Aschwanden, M. J., \& Malanushenko, A. 2013, SoPh, 287, 345

Aschwanden, M. J., \& Sandman, A. W. 2010, AJ, 140, 723

Aschwanden, M. J., Sun, X. D., \& Liu, Y. 2014a, ApJ, 785, 34

Aschwanden, M. J., Wuelser, J. P., Nitta, N. V., et al. 2012, ApJ, 756, 124

Aschwanden, M. J., Xu, Y., \& Jing, J. 2014b, ApJ, 797, 50

Berger, T. E., De Pontieu, B., Fletcher, L., et al. 1999, SoPh, 190, 409

Buehler, D., Lagg, A., Solanki, S. K., \& van Noort, M. 2015, A\&A, 576, 27

Carlsson, M., \& Leenaarts, J. 2012, A\&A, 539, A39

Carlsson, M., \& Stein, R. F. 1997, ApJ, 481, 500

Cauzzi, G., Reardon, K. P., Uitenbroek, H., et al. 2008, A\&A, 480, 515 Cavallini, F. 2006, SoPh, 236, 415

Choudhary, D. P., Sakurai, T., \& Venkatakrishnan, P. 2001, ApJ, 560, 439 de la Cruz Rodriguez, J., \& Socas-Navarro, H. 2011, A\&A, 527, L8

de la Cruz Rodriguez, J., Socas-Navarro, H., Carlsson, M., \& Leenaarts, J. 2012, A\&A, 543, A34

De Pontieu, B., Hansteen, V. H., Rouppe van der Voort, R., van Noort, M., \& Carlsson, M. 2007, in ASP Conf. Ser. 368, The Physics of Chromospheric Plasmas, ed. P. Heinzel, I. Dorotovic, \& R. J. Rutten (San Francisco, CA: ASP), 65

De Pontieu, B., Title, A. M., Lemen, J. R., et al. 2014, SoPh, 289, 2733

DeRosa, M. L., Schrijver, C. J., Barnes, G., et al. 2009, ApJ, 696, 1780

DeRosa, M. L., Wheatland, M. S., Leka, K. D., et al. 2015, ApJ, 811, 107

Ewell, W. M., Jr., Zirin, H., Jensen, J. B., \& Bastian, T. S. 1993, ApJ, 403, 426

Fontenla, J. M., Avrett, E. H., \& Loeser, R. 1990, ApJ, 355, 700

Fontenla, J. M., Avrett, E. H., \& Loeser, R. 1993, ApJ, 406, 319

Gabriel, A. H. 1976, RSPTA, 281, 339 
Gary, G. A. 2001, SoPh, 203, 71

Gudiksen, B. V., Carlsson, M., Hansteen, V. H., et al. 2011, A\&A, 531, A154 Harvey, J., Bippert-Plymate, T., Branston, D., et al. 1999, BAAS, 194, 94 Jendersie, S., \& Peter, H. 2006, A\&A, 460, 901

Jess, D. B., Mathioudakis, M., Christian, D. J., et al. 2010, SoPh, 261, 363

Jess, D. B., Morton, R. J., Verth, G., et al. 2015, SSRv, 190, 103

Jess, D. B., Reznikova, V. E., Van Doorsselaere, T., Keys, P. H., \& Mackay, D. H. 2013, ApJ, 779, 168

Jing, J., Yuan, Y., Reardon, K., et al. 2011, ApJ, 739, 67

Leenaarts, J., Carlsson, M., \& Rouppe van der Voort, L. 2012, ApJ, 749, 136

Leenaarts, J., Carlsson, M., Hansteen, V., \& Rouppe van der Voort, L. 2009, ApJL, 694, L128

Leenaarts, J., Carlsson, M., \& Rouppe van der Voort, L. 2015, ApJ, 802, 136

Lemen, J. R., Title, A. M., Akin, D. J., et al. 2012, SoPh, 275, 17

Malanushenko, A., Longcope, D. W., \& McKenzie, D. E. 2009, ApJ, 707, 1044

Malanushenko, A., Schrijver, C. J., DeRosa, M. L., \& Wheatland, M. S. 2014, ApJ, 783, 102

Malanushenko, A., Schrijver, C. J., DeRosa, M. L., Wheatland, M. S., \& Gilchrist, S. A. 2012, ApJ, 756, 153

Malanushenko, A., Yusuf, M. H., \& Longcope, D. W. 2011, ApJ, 736, 97

Metcalf, T. R., DeRosa, M. L., Schrijver, C. J., et al. 2008, SoPh, 247, 269

Metcalf, T. R., Jiao, L., Uitenbroek, H., McClymont, A. N., \& Canfield, R. C. 1995, ApJ, 439, 474

Molodenskii, M. M. 1969, SvA AJ, 12, 585
Pesnell, W. D., Thompson, B. J., \& Chamberlin, P. C. 2011, SoPh, 275, 3

Pietarila, A., Hirzberger, J., Zakharov, V., \& Solanki, S. K. 2009, A\&A, 502, 647

Reardon, K. P., \& Cavallini, F. 2008, A\&A, 481, 897

Reardon, K. P., Wang, Y. M., Muglach, K., \& Warren, H. P. 2011, ApJ, 742,119

Righini, A., Cavallini, F., \& Reardon, K. P. 2010, A\&A, 515, A85

Sandman, A. W., \& Aschwanden, M. J. 2011, SoPh, 270, 503

Sandman, A. W., Aschwanden, M. J., DeRosa, M. L., Wuelser, J. P., \& Alexander, D. 2009, SoPh, 259, 1

Schad, T. A., Penn, M. J., \& Lin, H. 2013, ApJ, 768, 111

Scharmer, G. B. 2006, A\&A, 447, 1111

Scherrer, P. H., Schou, J., Bush, R. I., et al. 2012, SoPh, 275, 207

Sedgewick, R. 2002, Algorithms in Java: Parts 1-4 (3rd ed.; Reading, MA: Addison-Wesley)

Socas-Navarro, H., Elmore, D., Pietarila, A., et al. 2006, SoPh, 235, 55

Valori, G., Kliem, B., \& Fuhrmann, M. 2007, SoPh, 245, 263

Valori, G., Kliem, B., Toeroek., T., \& Titov, S. 2010, A\&A, 519, A44

Vernazza, J. E., Avrett, E. H., \& Loeser, R. 1981, ApJSS, 45, 635

Wheatland, M. S. 2007, SoPh, 245, 251

Wheatland, M. S., Sturrock, P. A., \& Roumeliotis, G. 2000, ApJ, 540, 1150

Wiegelmann, T., \& Inhester, B. 2010, A\&A, 516, A107

Wiegelmann, T., Neukirch, T., Nickeler, D. H., et al. 2015, ApJ, 815, 10

Wiegelmann, T., Thalmann, J. K., Schrijver, C. J., DeRosa, M. L., \& Metcalf, T. R. 2008, SoPh, 247, 249 